\title{
Effects of one valence proton on seniority and angular momentum of neutrons in neutron-rich ${ }_{51}^{122-131} \mathrm{Sb}$ isotopes
}

S. Biswas, ${ }^{1, *}$ A. Lemasson,,${ }^{1, \dagger}$ M. Rejmund, ${ }^{1}$ A. Navin, ${ }^{1}$ Y. H. Kim, ${ }^{1, \star}$ C. Michelagnoli, ${ }^{1, \star}$ I. Stefan, ${ }^{2}$ R. Banik,${ }^{3,4}$ P. Bednarczyk, ${ }^{5}$ S. Bhattacharya, ${ }^{3,4}$ S. Bhattacharyya, ${ }^{3,4}$ E. Clément, ${ }^{1}$ H. L. Crawford, ${ }^{6}$ G. de France, ${ }^{1}$ P. Fallon, ${ }^{6}$ G. Frémont, ${ }^{1}$ J. Goupil,${ }^{1}$ B. Jacquot, ${ }^{1}$ H. J. Li,${ }^{1}$ J. Ljungvall, ${ }^{7}$ A. Maj,${ }^{5}$ L. Ménager, ${ }^{1}$ V. Morel, ${ }^{1}$ R. Palit, ${ }^{8}$ R. M. Pérez-Vidal,,${ }^{9}$ J. Ropert, ${ }^{1}$ D. Barrientos, ${ }^{10}$ G. Benzoni, ${ }^{11}$ B. Birkenbach, ${ }^{12}$ A. J. Boston, ${ }^{13}$ H. C. Boston, ${ }^{13}$ B. Cederwall,,${ }^{14}$ J. Collado, ${ }^{15}$ D. M. Cullen, ${ }^{16}$ P. Désesquelles, ${ }^{7}$ C. Domingo-Pardo,${ }^{9}$ J. Dudouet, ${ }^{7,17}$ J. Eberth,${ }^{12}$ V. González, ${ }^{15}$ L. J. Harkness-Brennan, ${ }^{13}$ H. Hess, ${ }^{12}$ A. Jungclaus, ${ }^{18}$ W. Korten, ${ }^{19}$ M. Labiche,${ }^{20}$ A. Lefevre, ${ }^{1}$ R. Menegazzo, ${ }^{21}$ D. Mengoni, ${ }^{21,22}$ B. Million, ${ }^{11}$ D. R. Napoli, ${ }^{23}$ A. Pullia, ${ }^{11,24}$ B. Quintana, ${ }^{25}$ D. Ralet, ${ }^{7,26,27}$ F. Recchia, ${ }^{21,22}$ P. Reiter, ${ }^{12}$ F. Saillant, ${ }^{1}$ M. D. Salsac, ${ }^{19}$ E. Sanchis,,${ }^{15}$ O. Stezowski, ${ }^{17}$ Ch. Theisen, ${ }^{19}$ J. J. Valiente-Dobón, ${ }^{23}$ and M. Zielińska ${ }^{19}$

${ }^{1}$ GANIL, CEA/DRF-CNRS/IN2P3, Bd Henri Becquerel, BP 55027, F-14076 Caen Cedex 5, France

${ }^{2}$ Institut de Physique Nucléaire, IN2P3-CNRS, Univ. Paris Sud, Université Paris Saclay, 91406 Orsay Cedex, France

${ }^{3}$ Variable Energy Cyclotron Centre, 1/AF Bidhan Nagar, Kolkata 700064, India

${ }^{4}$ Homi Bhabha National Institute, Training School Complex, Anushaktinagar, Mumbai-400094, India

${ }^{5}$ Institute of Nuclear Physics PAN, Kraków, Poland

${ }^{6}$ Nuclear Science Division, Lawrence Berkeley National Laboratory, Berkeley, California 94720, USA

${ }^{7}$ CSNSM, Univ. Paris-Sud, CNRS/IN2P3, Université Paris-Saclay, 91405 Orsay, France

${ }^{8}$ Department of Nuclear and Atomic Physics, Tata Institute of Fundamental Research, Mumbai 400005, India

${ }^{9}$ Instituto de Física Corpuscular, CSIC-Universitat de València, E-46980 València, Spain

${ }^{10} \mathrm{CERN}, \mathrm{CH}-1211$ Geneva 23 (Switzerland)

${ }^{11}$ INFN, Sezione di Milano, Milano, Italy

${ }^{12}$ Institut für Kernphysik, Universität zu Köln, Zülpicher Str. 77, D-50937 Köln, Germany

${ }^{13}$ Oliver Lodge Laboratory, The University of Liverpool, Liverpool, L69 7ZE, United Kingdom

${ }^{14}$ Department of Physics, Royal Institute of Technology, SE-10691 Stockholm, Sweden

${ }^{15}$ Departamento de Ingeniería Electrónica, Universitat de Valencia, Burjassot, Valencia, Spain

${ }^{16}$ Nuclear Physics Group, Schuster Laboratory, University of Manchester, Manchester, M13 9PL, United Kingdom

${ }^{17}$ Université de Lyon, Université Lyon-1, CNRS/IN2P3, UMR5822, IPNL, 4 Rue Enrico Fermi, F-69622 Villeurbanne Cedex, France

${ }^{18}$ Instituto de Estructura de la Materia, CSIC, Madrid, E-28006 Madrid, Spain

${ }^{19}$ IRFU, CEA/DRF, Centre CEA de Saclay, F-91191 Gif-sur-Yvette Cedex, France

${ }^{20}$ STFC Daresbury Laboratory, Daresbury, Warrington, WA4 4AD, United Kingdom

${ }^{21}$ INFN Sezione di Padova, I-35131 Padova, Italy

${ }^{22}$ Dipartimento di Fisica e Astronomia dell'Università di Padova, I-35131 Padova, Italy

${ }^{23}$ INFN, Laboratori Nazionali di Legnaro, Via Romea 4, I-35020 Legnaro, Italy

${ }^{24}$ Dipartimento di Fisica, Università di Milano, I-20133 Milano, Italy

${ }^{25}$ Laboratorio de Radiaciones Ionizantes, Universidad de Salamanca, E-37008 Salamanca, Spain

${ }^{26}$ Institut für Kernphysik, Technische Universität Darmstadt, D-64289 Darmstadt, Germany

${ }^{27}$ GSI, Helmholtzzentrum für Schwerionenforschung GmbH, D-64291 Darmstadt, Germany

(Received 15 March 2019; published 3 June 2019)

Background: Levels fulfilling the seniority scheme and relevant isomers are commonly observed features in semimagic nuclei; for example, in $\mathrm{Sn}$ isotopes $(Z=50)$. Seniority isomers in $\mathrm{Sn}$, with dominantly pure neutron configurations, directly probe the underlying neutron-neutron $(\nu v)$ interaction. Furthermore, an addition of a valence proton particle or hole, through neutron-proton $(v \pi)$ interaction, affects the neutron seniority as well as the angular momentum.

\footnotetext{
*biswas@ganil.fr

†lemasson@ganil.fr

${ }^{\ddagger}$ Present address: Institut Laue-Langevin, F-38042 Grenoble Cedex, France.
}

Published by the American Physical Society under the terms of the Creative Commons Attribution 4.0 International license. Further distribution of this work must maintain attribution to the author(s) and the published article's title, journal citation, and DOI. 
Purpose: Benchmark the reproducibility of the experimental observables, like the excitation energies $\left(E_{X}\right)$ and the reduced electric-quadrupole transition probabilities $[B(E 2)]$, with the results obtained from shell-model interactions for neutron-rich $\mathrm{Sn}$ and $\mathrm{Sb}$ isotopes with $N<82$. Study the sensitivity of the aforementioned experimental observables to the model interaction components. Furthermore, explore from a microscopic point of view the structural similarity between the isomers in $\mathrm{Sn}$ and $\mathrm{Sb}$, and thus the importance of the valence proton. Methods: The neutron-rich ${ }^{122-131} \mathrm{Sb}$ isotopes were produced as fission fragments in the reaction ${ }^{9} \mathrm{Be}\left({ }^{238} \mathrm{U}, f\right)$ with $6.2 \mathrm{MeV} / \mathrm{u}$ beam energy. A unique setup, consisting of AGATA, VAMOS ++ , and EXOGAM detectors, was used which enabled the prompt-delayed $\gamma$-ray spectroscopy of fission fragments in the time range of $100 \mathrm{~ns}$ to $200 \mu \mathrm{s}$.

Results: New isomers and prompt and delayed transitions were established in the even- $A{ }^{122-130} \mathrm{Sb}$ isotopes. In the odd- $A{ }^{123-131} \mathrm{Sb}$ isotopes, new prompt and delayed $\gamma$-ray transitions were identified, in addition to the confirmation of the previously known isomers. The half-lives of the isomeric states and the $B(E 2)$ transition probabilities of the observed transitions depopulating these isomers were extracted.

Conclusions: The experimental data was compared with the theoretical results obtained in the framework of large-scale shell-model (LSSM) calculations in a restricted model space. Modifications of several components of the shell-model interaction were introduced to obtain a consistent agreement with the excitation energies and the $B(E 2)$ transition probabilities in neutron-rich $\mathrm{Sn}$ and $\mathrm{Sb}$ isotopes. The isomeric configurations in $\mathrm{Sn}$ and $\mathrm{Sb}$ were found to be relatively pure. Furthermore, the calculations revealed that the presence of a single valence proton, mainly in the $g_{7 / 2}$ orbital in Sb isotopes, leads to significant mixing (due to the $\nu \pi$ interaction) of (i) the neutron seniorities $\left(v_{v}\right)$ and (ii) the neutron angular momentum $\left(I_{v}\right)$. The above features have a weak impact on the excitation energies, but have an important impact on the $B(E 2)$ transition probabilities. In addition, a constancy of the relative excitation energies irrespective of neutron seniority and neutron number in $\mathrm{Sn}$ and $\mathrm{Sb}$ was observed.

DOI: 10.1103/PhysRevC.99.064302

\section{INTRODUCTION}

The Sn isotopes, with basically a spherical shape, span between two doubly magic nuclei, the neutron-deficient ${ }^{100} \mathrm{Sn}$ $(Z=N=50)$ and the neutron-rich ${ }^{132} \mathrm{Sn}(Z=50, N=82)$. A constant trend, as a function of neutron number, in the excitation energies $\left(E_{X}\right)$ of the $2^{+}$state and the parabolic shape (with a dip around $N=66$ ) of the reduced electricquadrupole transition probabilities $\left[B\left(E 2 ; 2^{+} \rightarrow 0^{+}\right)\right]$, was observed for the even- $A \mathrm{Sn}$ isotopes $[1,2]$. The observed trend has recently been clarified by using state-of-the-art Monte Carlo shell-model (MCSM) calculations with a large model space, where the dip in the $B(E 2)$ was shown to correspond to a novel shape evolution from moderately deformed phase to pairing (seniority) phase around $N=66$ [3]. These calculations required huge computational resources, hence only $0^{+}, 2^{+}$, and $4^{+}$states were calculated for fitting purposes. Recently, the parabolic behavior of the $B\left(E 2 ; 10^{+} \rightarrow 8^{+}\right)$in even- $A{ }^{116-130} \mathrm{Sn}$ was described in the generalized seniority (v) scheme in Ref. [4], showing the necessity of the incorporation of configuration mixing of neutron $v h_{11 / 2}, v d_{3 / 2}$, and $\nu s_{1 / 2}$ orbitals instead of a pure $\nu h_{11 / 2}$ orbital, with generalized seniority being pure $v_{v}=2\left(v_{v}\right.$ stands for neutron seniority, which refers to the number of unpaired neutrons). It is known that seniority is a good quantum number for $l_{j} \leqslant f_{7 / 2}$. This becomes a partial symmetry in the case of $l_{j}=g_{9 / 2}$ in ${ }^{72,74} \mathrm{Ni}$ [5]. In these isotopes, the $\left(6^{+}\right)_{v_{\nu}=4}$ state has a lower excitation energy than the $\left(6^{+}\right)_{v_{v}=2}$, leading to a disappearance of the seniority $\left(8^{+}\right)_{v_{v}=2}$ isomers. At present, there is no indication of disappearance of $\left(10^{+}\right)_{v_{v}=2}$ seniority isomers in the $\mathrm{Sn}$ isotopes $\left(l_{j}=h_{11 / 2}\right)$, indicating that the $8^{+}$state in $\mathrm{Sn}$ is still a pure $v_{v}=2$ state. Recent results on the low-lying high-spin states in even- $A{ }^{124-128} \mathrm{Sb}(Z=51)$ and ${ }^{122-126}$ In $(Z=49)$ suggested that there is a significant mixing of the seniorities, $v_{v}=1$ and 3 , due to the presence of a single valence proton particle (hole) in the $g_{7 / 2}\left(g_{9 / 2}\right)$ orbital [6]. It was shown in Ref. [6] that the interaction of a single proton particle (hole) in the $g_{7 / 2}\left(g_{9 / 2}\right)$ spin-orbit partners in $\mathrm{Sb}(\mathrm{In})$ isotopes, respectively, with the neutrons energetically favored the state with one broken pair of neutrons (breaking of the seniority) over a state with no broken pairs for the low-lying high-spin states.

The experimental study of nuclei near the shell closures serves as an important test bench for directly probing the nucleon-nucleon interaction. This acts as important input for existing shell-model interactions and allows us to improve the predictive power of such calculations. In particular, the mass $A \sim 130$ region, near shell closure, is an interesting area of research in contemporary nuclear physics, because ${ }^{132} \mathrm{Sn}$ is so far the heaviest neutron-rich unstable doubly magic nucleus and it is possible to access this region with the recent advent of new accelerator facilities and large detector arrays [7-10]. The excitation energies $\left(E_{X}\right)$ and the transition strengths $[B(E 2) / B(M 1)]$ of neighboring nuclei near ${ }^{132} \mathrm{Sn}$ act as a testing ground to shell-model calculations and the associated interactions [6,11-15].

Even- $A{ }^{118-130} \mathrm{Sn}$ isotopes possess $7^{-}$and $10^{+}$isomers, with dominant neutron $v h_{11 / 2}^{-1} d_{3 / 2}^{-1}$ and $v h_{11 / 2}^{-2}$ configurations, respectively [16-19]. Similarly, odd- $A{ }^{119-129} \mathrm{Sn}$ isotopes display $23 / 2^{+}$and $27 / 2^{-}$isomers with dominant $\nu h_{11 / 2}^{-2} d_{3 / 2}^{-1}$ and $\nu h_{11 / 2}^{-3}$ configurations, respectively [18,20,21], an additional hole in the $v h_{11 / 2}$ orbital coupled to the counterparts, $7^{-}$and $10^{+}$isomers in even- $A$ Sn, respectively. The 
experimental data from these nuclei, due to proton shell closure, probe the neutron-neutron $(\nu v)$ interaction. Odd- $A$ ${ }^{121-131} \mathrm{Sb}$ isotopes have $19 / 2^{-}$and $23 / 2^{+}$isomers with dominant $\pi g_{7 / 2} \nu h_{11 / 2}^{-1} d_{3 / 2}^{-1}$ and $\pi g_{7 / 2} \nu h_{11 / 2}^{-2}$ configurations [22-26], an additional proton particle in $g_{7 / 2}$ coupled to the $7^{-}$and $10^{+}$isomers in even- $A \mathrm{Sn}$, respectively. The isomers in even- $A$ ${ }^{122-128} \mathrm{Sb}$ isotopes, except for ${ }^{130} \mathrm{Sb}$, were not known and are newly observed in this work. The experimental data on Sb isotopes, in addition to the information obtained for $\mathrm{Sn}$, probe the neutron-proton $(\nu \pi)$ interaction. The present paper discusses the four different cases of configurational similarities between $\mathrm{Sn}$ and $\mathrm{Sb}$ :

$$
\begin{aligned}
& \text { Case I: } 10^{+} \rightarrow 8^{+} \quad\left(v h_{11 / 2}^{-2}\right) \quad \text { in even- } A \quad \mathrm{Sn} \text { and } \\
& 23 / 2^{+} \rightarrow 19 / 2^{+}\left(\pi g_{7 / 2} \nu h_{11 / 2}^{-2}\right) \text { in odd- } A \mathrm{Sb} \text {. } \\
& \text { Case II: } 7^{-} \rightarrow 5^{-} \quad\left(v h_{11 / 2}^{-1} d_{3 / 2}^{-1}\right) \quad \text { in even- } A \quad \mathrm{Sn} \text { and } \\
& 19 / 2^{-} \rightarrow 15 / 2^{-} \quad\left(\pi g_{7 / 2} v h_{11 / 2}^{-1} d_{3 / 2}^{-1}\right) \text { in odd- } A \\
& \mathrm{Sb} \text {. } \\
& \text { Case III: } 23 / 2^{+} \rightarrow 19 / 2^{+}\left(v h_{11 / 2}^{-2} d_{3 / 2}^{-1}\right) \text { in odd- } A \text { Sn and } \\
& 13^{+} \rightarrow 11^{+}\left(\pi g_{7 / 2} v h_{11 / 2}^{-2} d_{3 / 2}^{-1}\right) \text { in even- } A \mathrm{Sb} \text {. } \\
& \text { Case IV: } 27 / 2^{-} \rightarrow 23 / 2^{-}\left(v h_{11 / 2}^{-3}\right) \text { in odd } A \text { Sn and } \\
& 16^{-} \rightarrow 14^{-}\left(\pi g_{7 / 2} v h_{11 / 2}^{-3}\right) \text { in even- } A \mathrm{Sb} \text {. }
\end{aligned}
$$

\section{EXPERIMENTAL DETAILS}

The neutron-rich ${ }^{122-131} \mathrm{Sb}$ isotopes were produced as fission fragments via fusion- and transfer-induced fission reactions at GANIL by using a ${ }^{238} \mathrm{U}$ beam at an energy of $6.2 \mathrm{MeV} / \mathrm{u}$, with typical beam intensity of $1 \mathrm{pnA}$ on a ${ }^{9} \mathrm{Be}$ target (1.6 and $5 \mu \mathrm{m}$ thick). The unambiguous isotopic identification of the fission fragments $(Z, A, q)$ was achieved using the VAMOS ++ spectrometer, placed at $20^{\circ}$ relative to the beam axis [27-29]. The focal plane detection system of VAMOS++ was constituted of a multiwire proportional counter (MWPC), two drift chambers, and a segmented ionization chamber. The AGATA $\gamma$-ray tracking array, consisting of 32 crystals, was placed at $13.5 \mathrm{~cm}$ from the target position [30]. The velocity vector of the recoiling ions [measured by a dual positionsensitive MWPC (DPS-MWPC) detector [31], placed at the entrance of the VAMOS ++ spectrometer], and the $\gamma$-ray emission angle (determined by using AGATA) were used to obtain the Doppler-corrected prompt $\gamma$ rays $\left(\gamma_{P}\right)$, on an eventby-event basis. Gamma-ray interaction points, determined by Pulse Shape Analysis (PSA) and GRID search algorithm techniques, were tracked by using the Orsay Forward Tracking (OFT) algorithm, as described in Ref. [32]. The delayed $\gamma$ rays $\left(\gamma_{D}\right)$ were detected by using seven EXOGAM HPGe Clover detectors [33], arranged in a wall-like configuration at the focal plane of VAMOS ++ . The particle identification (PID) spectra, intrinsic resolutions of the spectrometer $(\Delta Z / Z$, $\Delta A / A, \Delta q / q)$, and additional experimental details are given in Ref. [32].

The contamination from neighboring isobars, arising from the resolution in atomic number $Z$, was subtracted for prompt $\gamma$-ray spectra. In addition to the background reduction for the delayed spectra as suggested in Ref. [32], the subtraction in delayed spectra for neighboring isotopes was performed for all the $\mathrm{Sb}$ isotopes. The $\gamma-\gamma$ matrices $\left(\gamma_{P}-\gamma_{P}\right.$,
$\gamma_{D}-\gamma_{D}$, and $\left.\gamma_{P}-\gamma_{D}\right)$ were generated for both the prompt (P) and delayed (D) transitions for all the $\mathrm{Sb}$ isotopes. The uncertainties in the energy of the prompt and delayed $\gamma$ rays is $\sim 1 \mathrm{keV}$. The efficiencies for the prompt and delayed $\gamma$ rays were determined separately, as mentioned in Ref. [32]. The correction for the half-lives as mentioned in Ref. [32], was also taken into account. The spin-parities were assigned based on systematics and shell-model calculations. One- $\left(A_{0} e^{-t / A_{1} \ln 2}+A_{2}\right)$, two- $\left[A_{0} \frac{A_{2}}{A_{1}-A_{2}}\left(e^{-t / A_{1} \ln 2}-\right.\right.$ $\left.\left.e^{-t / A_{2} \ln 2}\right)+A_{3} e^{-t / A_{2} \ln 2}+A_{4}\right]$, and three-component fits, where $A_{i}(i=0, \ldots, 4)$ are the fitting parameters, were carried out for the estimation of half-lives of the states, wherever required. The conversion coefficients used for the estimation of the $B(E 2)$ transition probabilities were taken from BrIcc [34].

\section{EXPERIMENTAL RESULTS}

A summary of the level schemes for all the odd- $A$ and even- $A{ }^{122-131} \mathrm{Sb}$ isotopes are shown in Fig. 1. Already known $\gamma$-ray transitions are shown in black. Newly identified prompt and delayed transitions are shown by red and blue, respectively. Already known half-lives which have not been measured in this work are shown. The remeasured and newly measured half-lives have been indicated by a red line and a red box, respectively. The width of the arrows are proportional to the intensity of the transitions. The intensities of the prompt and delayed transitions have been separately measured and the lowest transition is normalized to 100 in each case. The prompt $\gamma$-ray transition emitted by the complementary fission fragment [28,29] (mainly from the fusion-fission) are observed and identified (marked by "c" in all the spectra) in the low-energy part of the $\gamma$-ray spectra. The random coincidences with $\mathrm{X}$-rays emitted by ${ }^{238} \mathrm{U}$ are also observed and are marked by an @ symbol.

\section{A. ${ }^{122} \mathrm{Sb}$}

Previous measurements on ${ }^{122} \mathrm{Sb}$ were reported in Refs. [35,36]. The level scheme as obtained in the present work is shown in Fig. 1. Table I shows the properties of all the transitions assigned in this work.

The $A$ - and $Z$-gated tracked Doppler-corrected prompt singles $\gamma$-ray $\left(\gamma_{P}\right)$ spectrum for ${ }^{122} \mathrm{Sb}$ is shown in Fig. 2 . Three new prompt transitions, 445, 657, and $1102 \mathrm{keV}$ transitions, are seen and marked with an asterisk. Based on the relative intensities, the $445 \mathrm{keV} \gamma$ ray is placed above the $(8)^{-}$ level (a $4.2 \mathrm{~min}$ isomeric state observed in Ref. [35]). In addition, it follows the systematics with the higher even- $A \mathrm{Sb}$ isotopes. Due to low statistics, no $\gamma_{P}-\gamma_{P}$ coincidence could be carried out. However, based on the summation of gammas, the intensities, and the systematics, the $657 \mathrm{keV}$ is placed above the $445 \mathrm{keV}$ with the $\left(10^{-}\right)$level decaying by the $1102 \mathrm{keV}$ to the $(8)^{-}$state. No $A$ - and $Z$-gated delayed $\gamma$ rays $\left(\gamma_{D}\right)$ in ${ }^{122} \mathrm{Sb}$ could be identified from the present experiment.

$$
\text { B. }{ }^{123} \mathrm{Sb}
$$

The spectroscopy of ${ }^{123} \mathrm{Sb}$ isotope was previously studied in Refs. [22,23,37,38]. The level scheme as obtained in the 


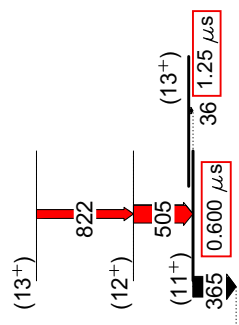
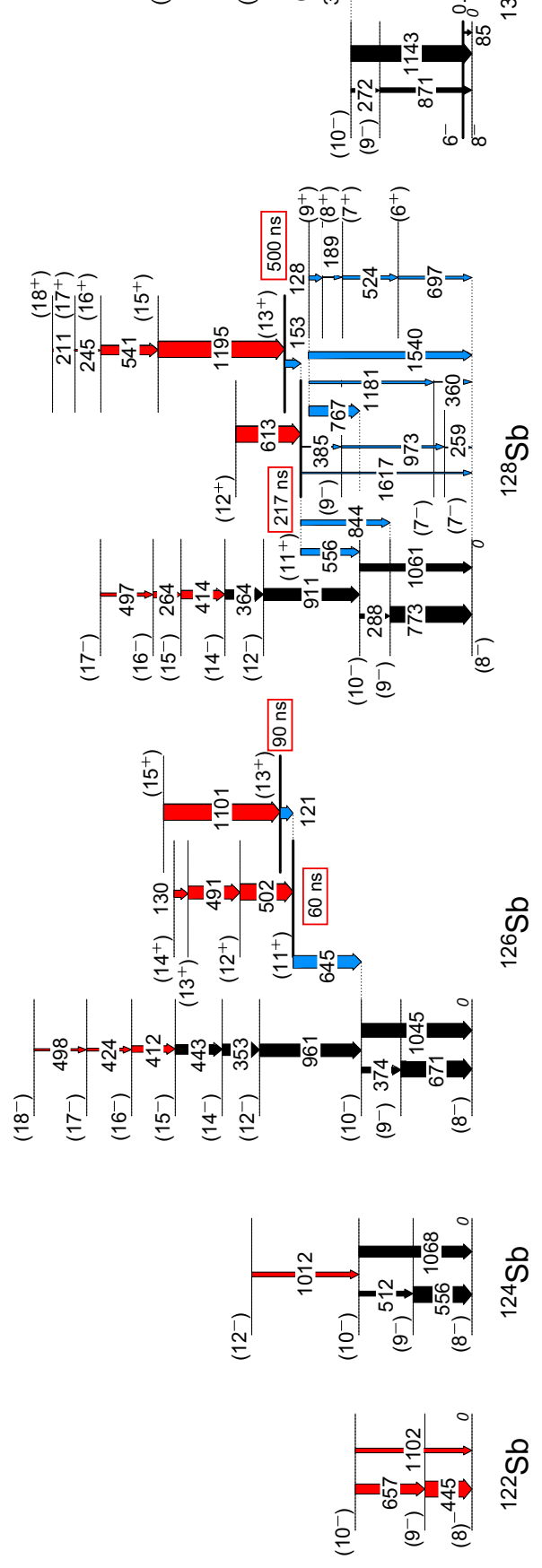
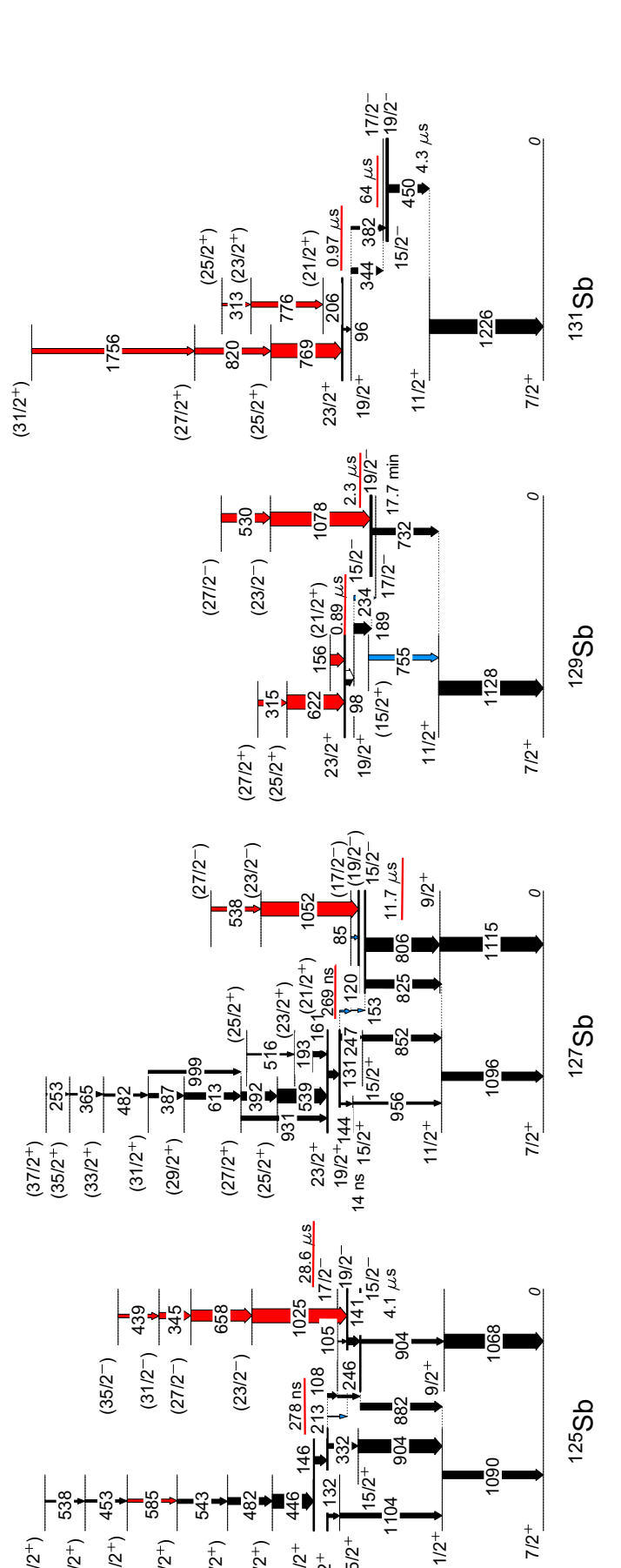

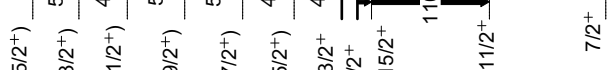

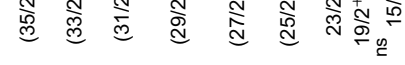

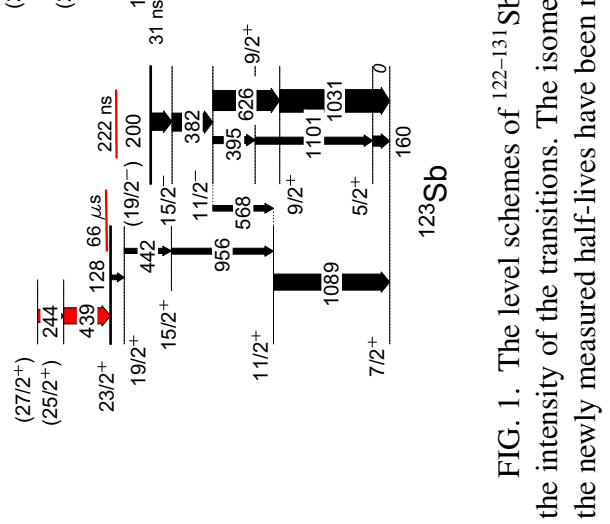




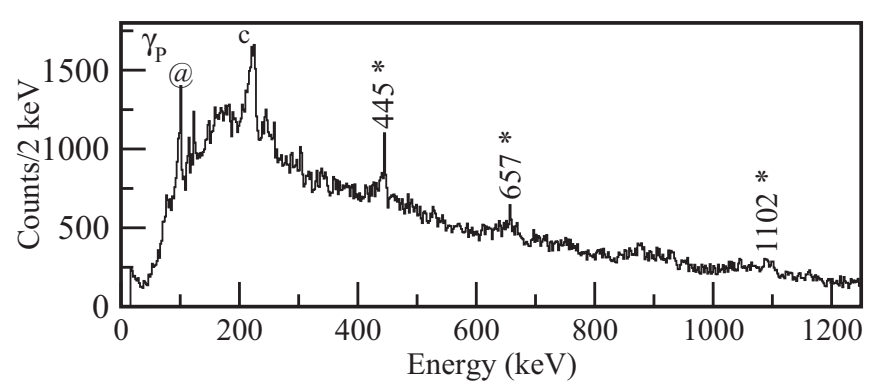

FIG. 2. $A$ - and $Z$-gated $\gamma$-ray spectrum for ${ }^{122} \mathrm{Sb}$ : The tracked Doppler-corrected prompt singles $\gamma$-ray $\left(\gamma_{P}\right)$ spectrum. The newly identified $\gamma$ rays are marked with an asterisk.

present work is shown in Fig. 1. Table II shows the properties of all the transitions assigned in this work.

The $A$ - and $Z$-gated $\gamma$-ray spectra for ${ }^{123} \mathrm{Sb}$ are shown in Fig. 3. The tracked Doppler-corrected prompt singles $\gamma$-ray spectrum $\left(\gamma_{P}\right)$ for ${ }^{123} \mathrm{Sb}$ is shown in Fig. 3(a). Previously observed 160,956 , and $1089 \mathrm{keV}$ transitions are seen in this spectrum. Two new prompt $\gamma$-ray transitions, namely 244 and $439 \mathrm{keV}$, are identified and these are marked with an asterisk. The delayed $\gamma$-ray spectrum $\left(\gamma_{D}\right)$ for $0<t_{\text {decay }}<2 \mu \mathrm{s}$ is shown in Fig. 3(b), yielding 160, 200, 382, 395, 568, 626, 1031, 1089, and $1101 \mathrm{keV}$ transitions, as expected from previous works. The half-life fit (one-component) for the decay spectrum upon gating on $200 \mathrm{keV}$ transition yields a value of $T_{1 / 2}=222(23) \mathrm{ns}$ for the $\left(19 / 2^{-}\right)$state [in agreement with the quoted value of 214(3) ns in Ref. [23]], which gives $B\left(E 2 ; 19 / 2^{-} \rightarrow 15 / 2^{-}\right)=6.9(7) e^{2} \mathrm{fm}^{4}$. However, the weak delayed transitions, which were mentioned in Refs. [23,38]; namely, the 949, 1007, 1260, and $1656 \mathrm{keV}$ transitions, are not observed owing to the low statistics in our dataset. Similarly, Fig. 3(c) shows the delayed $\gamma$-ray spectrum $\left(\gamma_{D}\right)$ for $6<t_{\text {decay }}<100 \mu \mathrm{s}$, leading to $128,442,956$, and $1089 \mathrm{keV}$ transitions. A half-life fit (one-component) for the decay spectrum upon gating on 128,442 , and $956 \mathrm{keV}$ transitions yields a value of $T_{1 / 2}=66(4) \mu$ s for the $23 / 2^{+}$state [in agreement with the value of 65(1) $\mu$ s reported in Ref. [23]], which gives $B\left(E 2 ; 23 / 2^{+} \rightarrow 19 / 2^{+}\right)=0.15(1) e^{2} \mathrm{fm}^{4}$. However, the weak transitions $100,148,348,375$, and $1250 \mathrm{keV}$, which were mentioned in Ref. [23], are not observed owing to the low statistics in our dataset. The tracked Doppler-corrected $\gamma_{P}$ in coincidence with any $\gamma_{D}$ (for $6<t_{\text {decay }}<100 \mu$ s) is shown in Fig. 3(d). This spectrum yields the two newly identified prompt $\gamma$-ray transitions, 244 and $439 \mathrm{keV}$ transitions. To confirm these newly found prompt transitions, a gate when applied on the prompt 244 and $439 \mathrm{keV}$ transitions yielded the delayed $128 \mathrm{keV}$ (not shown in this figure). Thus these two new transitions are placed above the $23 / 2^{+}$isomer. However,

TABLE I. Properties of the transitions assigned to ${ }^{122} \mathrm{Sb}$ obtained in this work.

\begin{tabular}{lcccc}
\hline \hline $\mathrm{E}_{\gamma}$ & $\mathrm{I}_{\gamma}$ & $J_{i}^{\pi} \rightarrow J_{f}^{\pi}$ & $E_{i}$ & $E_{f}$ \\
\hline 445.2 & 100 & $\left(9^{-}\right) \rightarrow(8)^{-}$ & 445 & 0 \\
657.2 & $63(14)$ & $\left(10^{-}\right) \rightarrow\left(9^{-}\right)$ & 1102 & 445 \\
1102.2 & $29(18)$ & $\left(10^{-}\right) \rightarrow(8)^{-}$ & 1102 & 0 \\
\hline \hline
\end{tabular}

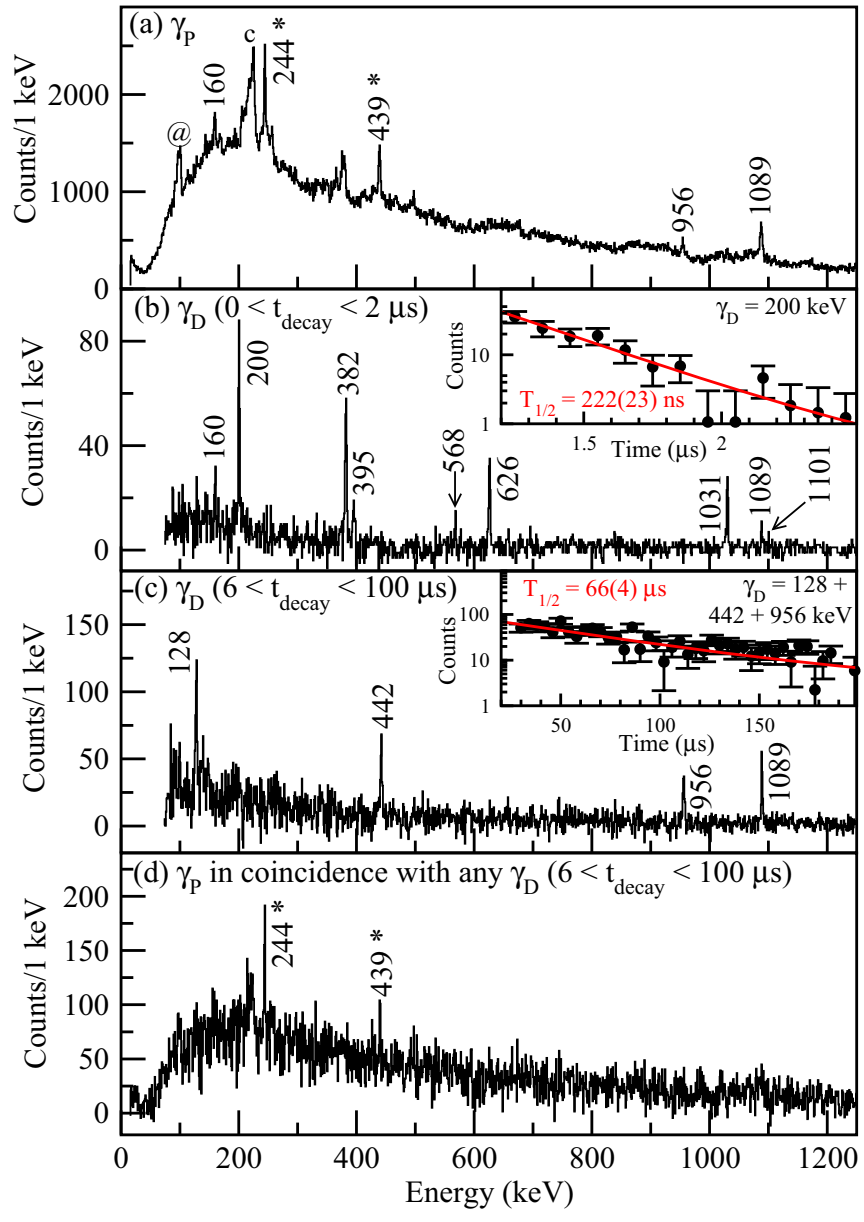

FIG. 3. $A$ - and $Z$-gated $\gamma$-ray spectra for ${ }^{123} \mathrm{Sb}$ : (a) The tracked Doppler-corrected prompt $\gamma$-ray $\left(\gamma_{P}\right)$ spectrum with the new $\gamma$-ray transitions marked with asterisk. (b), (c) The delayed $\gamma$-ray $\left(\gamma_{D}\right)$ spectra for $0<t_{\text {decay }}<2 \mu$ s and $6<t_{\text {decay }}<100 \mu$ s, respectively. The insets in panels (b) and (c) show the decay curves along with the fits for the 200 and $128+442+956 \mathrm{keV}$ transitions, respectively. (d) $\gamma_{P}$ in coincidence with any $\gamma_{D}$ for $6<t_{\text {decay }}<100 \mu \mathrm{s}$.

TABLE II. Properties of the transitions assigned to ${ }^{123} \mathrm{Sb}$ obtained in this work. The top and bottom panels, separated by a line, are for the prompt and delayed transitions, respectively.

\begin{tabular}{lcccc}
\hline \hline $\mathrm{E}_{\gamma}$ & $\mathrm{I}_{\gamma}$ & $J_{i}^{\pi} \rightarrow J_{f}^{\pi}$ & $E_{i}$ & $E_{f}$ \\
\hline 244.1 & $78(16)$ & $\left(27 / 2^{+}\right) \rightarrow\left(25 / 2^{+}\right)$ & 3298 & 3054 \\
439.2 & 100 & $\left(25 / 2^{+}\right) \rightarrow 23 / 2^{+}$ & 3054 & 2615 \\
\hline 128.2 & $29(16)$ & $23 / 2^{+} \rightarrow 19 / 2^{+}$ & 2615 & 2487 \\
159.8 & $63(36)$ & $5 / 2^{+} \rightarrow 7 / 2^{+}$ & 160 & 0 \\
200.4 & $108(57)$ & $\left(19 / 2^{-}\right) \rightarrow 15 / 2^{-}$ & 2239 & 2039 \\
382.0 & $112(54)$ & $15 / 2^{-} \rightarrow 11 / 2^{-}$ & 2039 & 1657 \\
395.4 & $44(23)$ & $11 / 2^{-} \rightarrow 9 / 2^{+}$ & 1657 & 1261 \\
441.6 & $32(11)$ & $19 / 2^{+} \rightarrow 15 / 2^{+}$ & 2487 & 2045 \\
568.2 & $35(17)$ & $11 / 2^{-} \rightarrow 11 / 2^{+}$ & 1657 & 1089 \\
626.0 & $125(56)$ & $11 / 2^{-} \rightarrow 9 / 2^{+}$ & 1657 & 1031 \\
956.0 & $37(14)$ & $15 / 2^{+} \rightarrow 11 / 2^{+}$ & 2045 & 1089 \\
1030.5 & $141(64)$ & $9 / 2^{+} \rightarrow 7 / 2^{+}$ & 1031 & 0 \\
1089.0 & 100 & $11 / 2^{+} \rightarrow 7 / 2^{+}$ & 1089 & 0 \\
1101.0 & $44(22)$ & $9 / 2^{+} \rightarrow 5 / 2^{+}$ & 1261 & 160 \\
\hline \hline
\end{tabular}




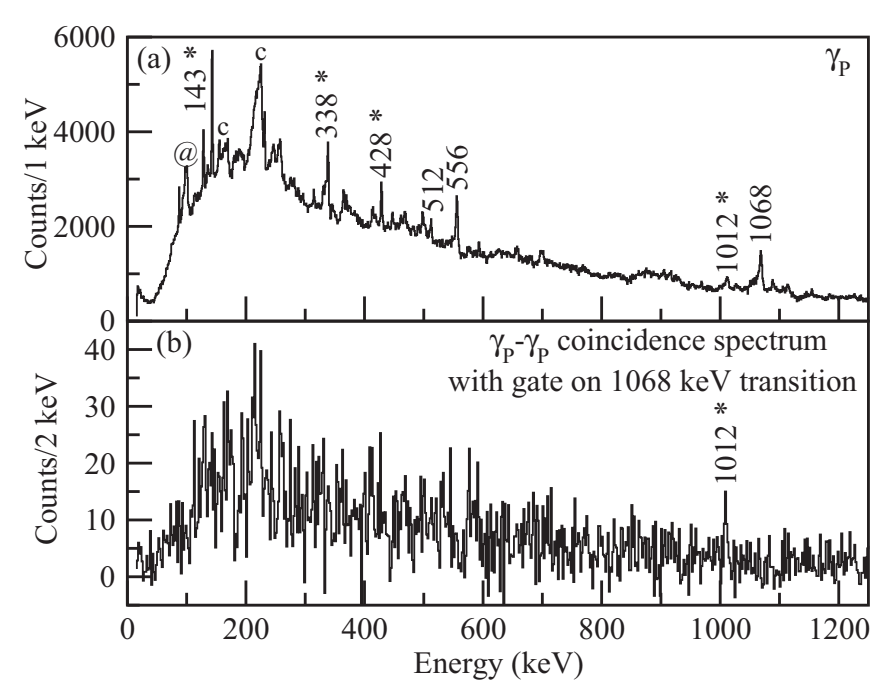

FIG. 4. $A$ - and $Z$-gated $\gamma$-ray spectra for ${ }^{124} \mathrm{Sb}$ : (a) The tracked Doppler-corrected prompt $\gamma$-ray $\left(\gamma_{P}\right)$ singles spectrum. (b) The prompt $\gamma-\gamma\left(\gamma_{P}-\gamma_{P}\right)$ spectrum with gate on $1068 \mathrm{keV}$ transition. The newly identified prompt transitions are marked with an asterisk.

the tracked Doppler-corrected $\gamma_{P}$ in coincidence with any $\gamma_{D}$ for $0<t_{\text {decay }}<2 \mu \mathrm{s}$, did not result in any new prompt $\gamma$ rays and hence no $\gamma$ ray is placed above the $\left(19 / 2^{-}\right)$isomer.

$$
\text { C. }{ }^{124} \mathrm{Sb}
$$

The $\gamma$-ray spectroscopy measurement on the high-spin states in ${ }^{124} \mathrm{Sb}$ was previously reported in Ref. [6]. The level scheme as obtained in the present work is shown in Fig. 1. Table III shows the properties of all the transitions assigned in this work.

The $A$ - and $Z$-gated tracked Doppler-corrected prompt singles $\gamma$-ray spectrum for ${ }^{124} \mathrm{Sb}$ is shown in Fig. 4(a). The already identified $\gamma$ rays in Ref. [6]; namely 512, 556, and $1068 \mathrm{keV}$ are seen. Other than these, four new prompt $143,338,428$, and $1012 \mathrm{keV} \gamma$-ray transitions are identified (marked with an asterisk). Figure 4(b) shows the $A$ - and $Z$-gated tracked Doppler-corrected prompt $\gamma-\gamma$ coincidence $\left(\gamma_{P}-\gamma_{P}\right)$ spectrum with gate on the $1068 \mathrm{keV}$ transition. This shows that the $1012 \mathrm{keV}$ transition is in coincidence and hence is placed in the level scheme. The placement of $1012 \mathrm{keV}$ transition in the level scheme follows the systematics with the other even- $A \mathrm{Sb}$ isotopes. The other prompt transitions 143, 338 , and $428 \mathrm{keV}$ are not placed as these are not observed in the coincidence spectrum. These $\gamma$ rays probably belong to the sideband (as observed above the isomer in the case of

TABLE III. Properties of the transitions assigned to ${ }^{124} \mathrm{Sb}$ obtained in this work.

\begin{tabular}{lcccc}
\hline \hline $\mathrm{E}_{\gamma}$ & $\mathrm{I}_{\gamma}$ & $J_{i}^{\pi} \rightarrow J_{f}^{\pi}$ & $E_{i}$ & $E_{f}$ \\
\hline 512.3 & $30(6)$ & $\left(10^{-}\right) \rightarrow\left(9^{-}\right)$ & 1068 & 556 \\
556.0 & 100 & $\left(9^{-}\right) \rightarrow\left(8^{-}\right)$ & 556 & 0 \\
1012.0 & $29(6)$ & $\left(12^{-}\right) \rightarrow\left(10^{-}\right)$ & 2080 & 1068 \\
1068.4 & $69(13)$ & $\left(10^{-}\right) \rightarrow\left(8^{-}\right)$ & 1068 & 0 \\
\hline \hline
\end{tabular}

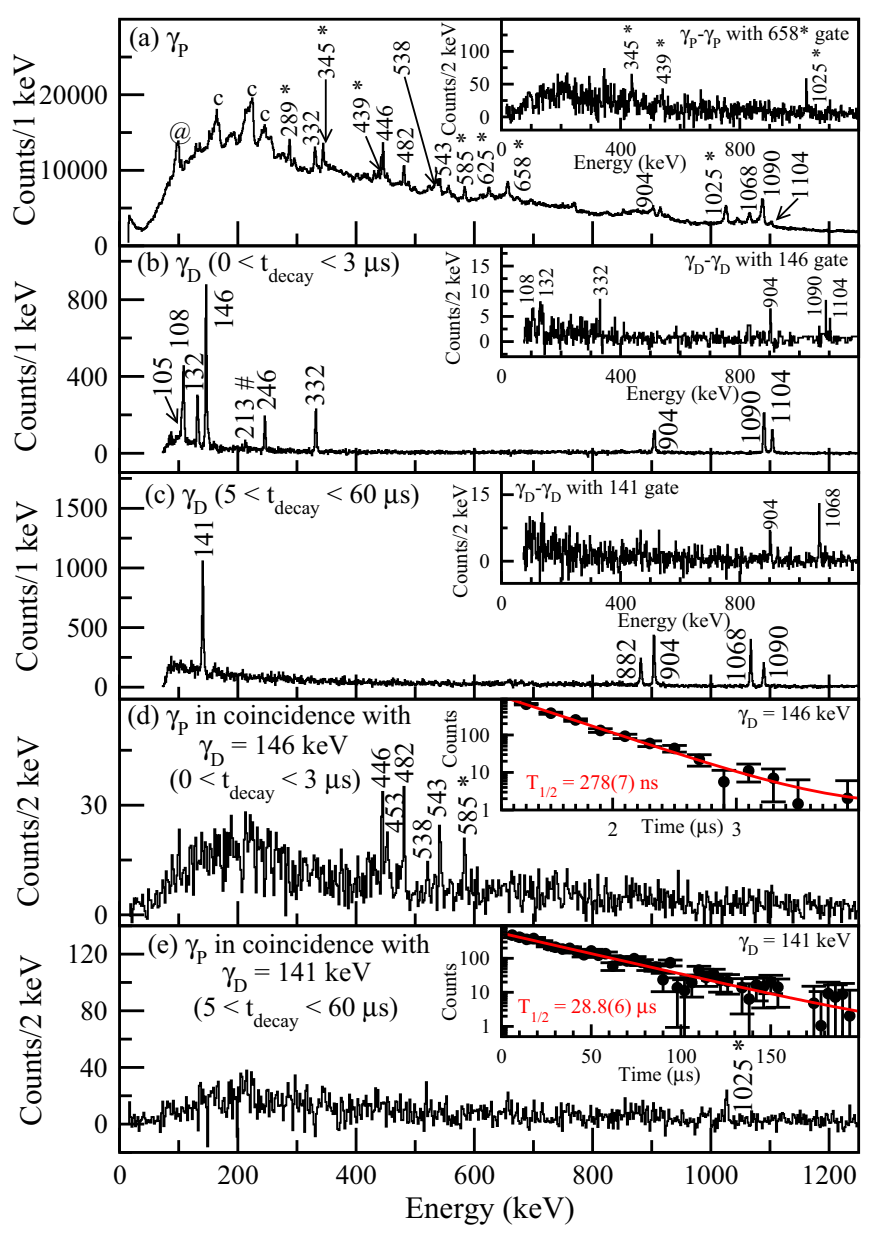

FIG. 5. $A$ - and $Z$-gated $\gamma$-ray spectra for ${ }^{125} \mathrm{Sb}$ : (a) The tracked Doppler-corrected prompt singles $\gamma$-ray $\left(\gamma_{P}\right)$ spectrum with the new $\gamma$-ray transitions marked with asterisk. The inset shows the tracked Doppler-corrected prompt $\gamma_{P}-\gamma_{P}$ coincidence spectrum with gate on the newly observed $658 \mathrm{keV} \gamma$-ray. (b), (c) The delayed singles $\gamma$-ray $\left(\gamma_{D}\right)$ spectra for $0<t_{\text {decay }}<3 \mu \mathrm{s}$ and $5<t_{\text {decay }}<60 \mu \mathrm{s}$, respectively. The insets in panels (b) and (c) shows the delayed $\gamma_{D}-\gamma_{D}$ coincidence spectra with gate on 146 and $141 \mathrm{keV} \gamma$ rays, respectively. (d) Tracked Doppler-corrected $\gamma_{P}$ in coincidence with $\gamma_{D}=146 \mathrm{keV} \gamma$-ray (for $0<t_{\text {decay }}<3 \mu \mathrm{s}$ ). The inset shows the decay curve along with the fit for the $146 \mathrm{keV}$ transition. (e) $\gamma_{P}$ in coincidence with the $\gamma_{D}=141 \mathrm{keV} \gamma$-ray (for $5<t_{\text {decay }}<60 \mu \mathrm{s}$ ). The inset shows the decay curve along with the fit for the $141 \mathrm{keV}$ transition.

${ }^{126} \mathrm{Sb}$ ). No delayed $\gamma$ rays could be identified for ${ }^{124} \mathrm{Sb}$, using the present setup.

$$
\text { D. }{ }^{125} \mathrm{Sb}
$$

Previous measurements on the $\gamma$-ray spectroscopy of highspin states in ${ }^{125} \mathrm{Sb}$ were reported in Refs. [22,37,39]. The level scheme as obtained in the present work is shown in Fig. 1. Table IV shows the properties of all the transitions assigned in this work.

The $A$ - and $Z$-gated $\gamma$-ray spectra for ${ }^{125} \mathrm{Sb}$ is shown in Fig. 5. The tracked Doppler-corrected prompt singles $\gamma$-ray spectrum $\left(\gamma_{P}\right)$ for ${ }^{125} \mathrm{Sb}$ is shown in Fig. 5(a). The previously 
TABLE IV. Properties of the transitions assigned to ${ }^{125} \mathrm{Sb}$ obtained in this work. The top and bottom panels, separated by a line, are for the prompt and delayed transitions, respectively.

\begin{tabular}{lcccr}
\hline \hline $\mathrm{E}_{\gamma}$ & $\mathrm{I}_{\gamma}$ & $J_{i}^{\pi} \rightarrow J_{f}^{\pi}$ & $E_{i}$ & $E_{f}$ \\
\hline 345.3 & $45(5)$ & $\left(31 / 2^{-}\right) \rightarrow\left(27 / 2^{-}\right)$ & 4138 & 3795 \\
438.7 & $26(3)$ & $\left(35 / 2^{-}\right) \rightarrow\left(31 / 2^{-}\right)$ & 4579 & 4138 \\
446.5 & 100 & $\left(25 / 2^{+}\right) \rightarrow 23 / 2^{+}$ & 2919 & 2472 \\
453.1 & $17(2)$ & $\left(33 / 2^{+}\right) \rightarrow\left(31 / 2^{+}\right)$ & 4981 & 4528 \\
482.0 & $50(3)$ & $\left(27 / 2^{+}\right) \rightarrow\left(25 / 2^{+}\right)$ & 3401 & 2919 \\
538.1 & $13(3)$ & $\left(35 / 2^{+}\right) \rightarrow\left(33 / 2^{+}\right)$ & 5366 & 4934 \\
542.7 & $30(2)$ & $\left(29 / 2^{+}\right) \rightarrow\left(27 / 2^{+}\right)$ & 3943 & 3401 \\
584.8 & $23(3)$ & $\left(31 / 2^{+}\right) \rightarrow\left(29 / 2^{+}\right)$ & 4528 & 3943 \\
657.8 & $79(6)$ & $\left(27 / 2^{-}\right) \rightarrow\left(23 / 2^{-}\right)$ & 3795 & 3138 \\
1025.4 & 100 & $\left(23 / 2^{-}\right) \rightarrow 19 / 2^{-}$ & 3138 & 2112 \\
\hline 105.1 & $6(4)$ & $17 / 2^{-} \rightarrow 19 / 2^{-}$ & 2217 & 2112 \\
108.1 & $29(19)$ & $19 / 2^{+} \rightarrow 17 / 2^{-}$ & 2326 & 2217 \\
132.1 & $24(13)$ & $19 / 2^{+} \rightarrow 15 / 2^{+}$ & 2326 & 2194 \\
140.7 & $51(28)$ & $19 / 2^{-} \rightarrow 15 / 2^{-}$ & 2112 & 1972 \\
146.3 & $52(27)$ & $23 / 2^{+} \rightarrow 19 / 2^{+}$ & 2472 & 2326 \\
213.3 & $5(2)$ & $19 / 2^{+} \rightarrow 19 / 2^{-}$ & 2326 & 2112 \\
245.9 & $19(11)$ & $17 / 2^{-} \rightarrow 15 / 2^{-}$ & 2217 & 1972 \\
332.0 & $34(13)$ & $19 / 2^{+} \rightarrow 15 / 2^{+}$ & 2326 & 1994 \\
881.8 & $50(17)$ & $15 / 2^{-} \rightarrow 11 / 2^{+}$ & 1972 & 1090 \\
903.8 & $95(32)$ & $15 / 2^{-} \rightarrow 9 / 2^{+}$ & 1972 & 1068 \\
904.1 & $29(10)$ & $15 / 2^{+} \rightarrow 11 / 2^{+}$ & 1994 & 1090 \\
1067.7 & 100 & $9 / 2^{+} \rightarrow 7 / 2^{+}$ & 1068 & 0 \\
1089.7 & $54(18)$ & $11 / 2^{+} \rightarrow 7 / 2^{+}$ & 1090 & 0 \\
1104.1 & $35(12)$ & $15 / 2^{+} \rightarrow 11 / 2^{+}$ & 2194 & 1090 \\
\hline \hline & & & & \\
\hline & & & &
\end{tabular}

known 332, 446, 482, 453, 538, 543, 904, 1068, 1090, and $1104 \mathrm{keV}$ transitions are observed. Seven new prompt $\gamma$ ray transitions, namely $289,345,439,585,625,658$, and $1025 \mathrm{keV}$ transitions are identified (marked with an asterisk). The inset shows the tracked Doppler-corrected prompt $\gamma_{P}-\gamma_{P}$ coincidence spectrum with gate on the newly identified $658 \mathrm{keV}$ transition. This spectrum shows that the 345, 439, 658 , and $1025 \mathrm{keV} \gamma$ rays are in coincidence. A similar coincidence spectrum was obtained for the 446, 453, 482, 538, 543 , and $585 \mathrm{keV}$ transitions (not shown in this figure). No $\gamma$ rays are seen in coincidence with the 289 and $625 \mathrm{keV}$ transitions, hence these are not placed in the level scheme. The delayed $\gamma$-ray $\left(\gamma_{D}\right)$ spectrum for $0<t_{\text {decay }}<3 \mu \mathrm{s}$ is shown in Fig. 5(b) yielding 105, 108, 132, 146, 213, 246, 332, $904\left(15 / 2^{+} \rightarrow 11 / 2^{+}\right), 1090$, and $1104 \mathrm{keV}$ transitions, as expected from the level scheme. The $213 \mathrm{keV}$ is newly identified and this is marked with a hash in the spectrum. The inset of Fig. 5(b) shows the delayed $\gamma_{D^{-}} \gamma_{D}$ coincidence spectrum with gate on $146 \mathrm{keV}$ transition, yielding all the intense delayed transitions. Similarly, Fig. 5(c) shows the delayed $\gamma$-ray spectrum for $5<t_{\text {decay }}<60 \mu$ s, leading to $141,882,904\left(15 / 2^{-} \rightarrow 9 / 2^{+}\right), 1068$, and $1090 \mathrm{keV}$ transitions. The inset of Fig. 5(c) shows the delayed $\gamma_{D}-\gamma_{D}$ coincidence spectrum with gate on $141 \mathrm{keV}$ transition, yielding 904 and $1068 \mathrm{keV} \gamma$ rays, as expected from previous works. The tracked Doppler-corrected $\gamma_{P}$ in coincidence with $\gamma_{D}=146 \mathrm{keV}$ (for $0<t_{\text {decay }}<3 \mu \mathrm{s}$ ) is shown in Fig. 5(d). This spectrum yields the prompt $\gamma$-ray transitions, 446, 453,
482, 538, 543, and $585 \mathrm{keV}$ transitions. In the previous Ref. [37], these prompt transitions, except $585 \mathrm{keV}$, were assigned negative-parities and placed above the 19/2- isomer. Here we propose these transitions to be positive-parity states and placed above the $23 / 2^{+}$isomer, as observed from promptdelayed coincidences. Based on the intensities, the $585 \mathrm{keV}$ transition is placed above the $543 \mathrm{keV}$ transition, and the 453 and $538 \mathrm{keV}$ transitions are replaced. The $432 \mathrm{keV}$ transition, seen in Ref. [37], is not observed in coincidence, hence it is not assigned in the present level scheme. The half-life fit (one-component) for the decay spectrum upon gating on $146 \mathrm{keV}$ transition yields a value of $T_{1 / 2}=278(7) \mathrm{ns}$ for the $23 / 2^{+}$[in agreement with the value of $272(16)$ ns reported in Ref. [22]], which is shown in the inset of Fig. 5(d). This leads to $B\left(E 2 ; 23 / 2^{+} \rightarrow 19 / 2^{+}\right)=21.3(6) e^{2} \mathrm{fm}^{4}$. The tracked Doppler-corrected $\gamma_{P}$ in coincidence with $\gamma_{D}=141 \mathrm{keV}$ for $5<t_{\text {decay }}<60 \mu$ s resulted in the observation of the newly identified $1025 \mathrm{keV} \gamma$ ray. As seen from the inset of Fig. 5(a), the 345, 439, 658, and $1025 \mathrm{keV}$ transitions are in coincidence, and hence are placed above the $19 / 2^{-}$ isomer. The inset of Fig. 5(d) shows the half-life fit (twocomponent with one component fixed to $278 \mathrm{~ns}$ ) for the decay spectrum upon gating on $141 \mathrm{keV}$ transition yield a value of $T_{1 / 2}=28.8(6) \mu$ s for the $19 / 2^{-}$state [in agreement with the quoted value of 28.0(7) $\mu$ s in Ref. [22]], which yields $B\left(E 2 ; 19 / 2^{-} \rightarrow 15 / 2^{-}\right)=0.24(1) e^{2} \mathrm{fm}^{4}$.

$$
\text { E. }{ }^{126} \mathrm{Sb}
$$

Gamma-ray spectroscopy measurements on the high-spin states in ${ }^{126} \mathrm{Sb}$ was previously reported in Ref. [6]. The level scheme as obtained in the present work is shown in Fig. 1. Table $\mathrm{V}$ shows the properties of all the transitions assigned in this work.

The $A$ - and $Z$-gated $\gamma$-ray spectra for ${ }^{126} \mathrm{Sb}$ are shown in Fig. 6. The tracked Doppler-corrected prompt singles $\gamma$-ray spectrum $\left(\gamma_{P}\right)$ for ${ }^{126} \mathrm{Sb}$ is shown in Fig. 6(a). The already known $\gamma$ rays from Ref. [6]; namely, 353, 374, 443, 671, 961, and $1045 \mathrm{keV}$ are observed. In addition, new prompt $\gamma$ rays $130,412,424,491,498,502$, and $1101 \mathrm{keV}$ (marked with an asterisk) are identified. The tracked Doppler-corrected prompt $\gamma-\gamma$ coincidence $\left(\gamma_{P}-\gamma_{P}\right)$ spectrum with a sum gate of 353 , 443 and $961 \mathrm{keV}$ prompt $\gamma$-ray transitions is shown in the inset of Fig. 6(a). This spectrum shows that the 353, 374, 412, $424,443,498,671,961$, and $1045 \mathrm{keV}$ transitions are in coincidence and the newly observed transitions are marked with an asterisk. Figure 6(b) shows the delayed $\gamma$-ray $\left(\gamma_{D}\right)$ singles spectrum, with $0<t_{\text {decay }}<4 \mu \mathrm{s}$. This spectrum shows the presence of two new delayed 121 and $645 \mathrm{keV}$ transitions, besides the $1045 \mathrm{keV}$ transition (marked with a hash). The inset in Fig. 6(b) shows the tracked Doppler-corrected prompt $\gamma-\gamma$ coincidence spectrum $\left(\gamma_{P}-\gamma_{P}\right)$ gated on the newly identified $491 \mathrm{keV}$ transition. This shows that the 130, 491, and $502 \mathrm{keV}$ transitions are in coincidence and these are placed above the $\left(11^{+}\right)$state in the level scheme, following the systematics with higher even- $A \mathrm{Sb}$ isotopes. Also in Fig. 6(a), the newly identified $1101 \mathrm{keV}$ transition, is not observed in the tracked Doppler-corrected prompt $\gamma_{P}-\gamma_{P}$ spectra [insets of Fig. 6(a) and $6(\mathrm{~b})]$. Following the systematics with ${ }^{128} \mathrm{Sb}$, this is placed 

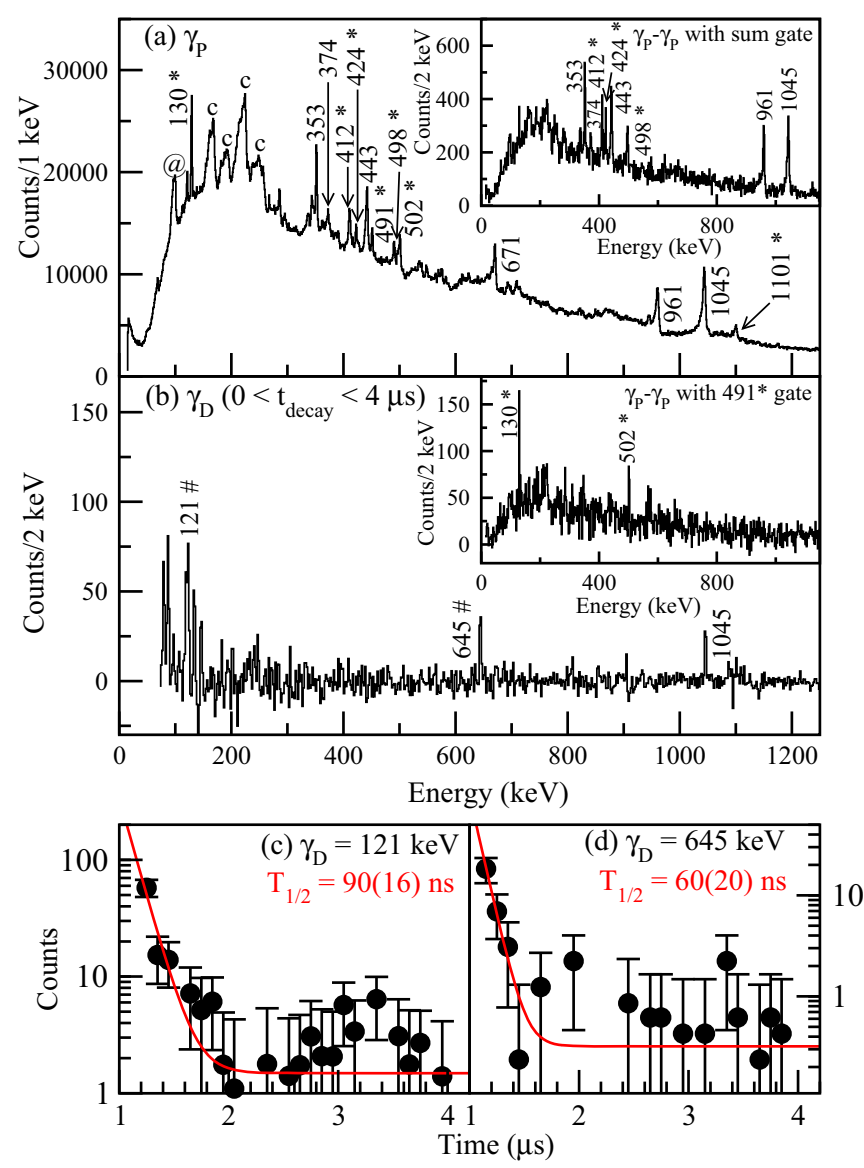

FIG. 6. $A$ - and $Z$-gated $\gamma$-ray spectra for ${ }^{126} \mathrm{Sb}$ : (a) Tracked Doppler-corrected prompt $\gamma$-ray singles spectrum $\left(\gamma_{P}\right)$. The inset shows the tracked Doppler-corrected $\gamma_{P}-\gamma_{P}$ coincidence spectrum with sum gate on $\gamma_{P} \mathrm{~s}$; namely, the 353,443 , and $961 \mathrm{keV}$ transitions. The newly identified prompt $\gamma$ rays are marked with an asterisk. (b) The delayed singles $\gamma$-ray spectrum $\left(\gamma_{D}\right)$ for $0<t_{\text {decay }}<4 \mu \mathrm{s}$. The newly identified delayed $\gamma$ rays are marked with a hash. The inset shows the tracked Doppler-corrected $\gamma_{P}-\gamma_{P}$ coincidence spectrum with gate on the newly identified $491 \mathrm{keV}$ prompt $\gamma$ ray. (c), (d) The decay curve along with the fit for the delayed 121 and $645 \mathrm{keV}$ transitions, respectively.

above the $\left(13^{+}\right)$state. The decay spectrum of the delayed $121 \mathrm{keV}$ transition is shown in Fig. 6(c). An exponential fit (one-component) yields a value of 90(16) ns. This gives $B\left(E 2 ; 13^{+} \rightarrow 11^{+}\right)=131(28) e^{2} \mathrm{fm}^{4}$. The half-life of the $645 \mathrm{keV}$ transition was also measured with $T_{1 / 2}=60(20) \mathrm{ns}$, as shown in Fig. 6(d) (using a two-component fit with one component fixed to $90 \mathrm{~ns}$ ).

\section{F. ${ }^{127} \mathrm{Sb}$}

Previous $\gamma$-ray spectroscopy measurements on ${ }^{127} \mathrm{Sb}$ were reported in Refs. [24,37,40]. The level scheme as obtained in the present work is shown in Fig. 1. Table VI shows the properties of all the transitions assigned in this work.

The $A$ - and $Z$-gated $\gamma$-ray spectra for ${ }^{127} \mathrm{Sb}$ is shown in Fig. 7. The tracked Doppler-corrected prompt singles $\gamma$-ray spectrum $\left(\gamma_{P}\right)$ for ${ }^{127} \mathrm{Sb}$ is shown in Fig. 7(a). The previously
TABLE V. Properties of the transitions assigned to ${ }^{126} \mathrm{Sb}$ obtained in this work. The top and bottom panels, separated by a line, are for the prompt and delayed transitions, respectively.

\begin{tabular}{lcccc}
\hline \hline $\mathrm{E}_{\gamma}$ & $\mathrm{I}_{\gamma}$ & $J_{i}^{\pi} \rightarrow J_{f}^{\pi}$ & $E_{i}$ & $E_{f}$ \\
\hline 130.1 & $44(4)$ & $\left(14^{+}\right) \rightarrow\left(13^{+}\right)$ & 2813 & 2682 \\
352.9 & $72(7)$ & $\left(14^{-}\right) \rightarrow\left(12^{-}\right)$ & 2359 & 2006 \\
373.5 & $35(5)$ & $\left(10^{-}\right) \rightarrow\left(9^{-}\right)$ & 1045 & 671 \\
411.9 & $39(5)$ & $\left(16^{-}\right) \rightarrow\left(15^{-}\right)$ & 3214 & 2802 \\
423.5 & $17(2)$ & $\left(17^{-}\right) \rightarrow\left(16^{-}\right)$ & 3638 & 3214 \\
443.3 & $59(7)$ & $\left(15^{-}\right) \rightarrow\left(14^{-}\right)$ & 2802 & 2359 \\
491.4 & $78(7)$ & $\left(13^{+}\right) \rightarrow\left(12^{+}\right)$ & 2682 & 2191 \\
497.6 & $13(2)$ & $\left(18^{-}\right) \rightarrow\left(17^{-}\right)$ & 4135 & 3638 \\
501.8 & 100 & $\left(12^{+}\right) \rightarrow\left(11^{+}\right)$ & 2191 & 1689 \\
671.2 & 100 & $\left(9^{-}\right) \rightarrow\left(8^{-}\right)$ & 671 & 0 \\
961.4 & $79(11)$ & $\left(12^{-}\right) \rightarrow\left(10^{-}\right)$ & 2006 & 1045 \\
1044.9 & $87(7)$ & $\left(10^{-}\right) \rightarrow\left(8^{-}\right)$ & 1045 & 0 \\
1101.1 & 100 & $\left(15^{+}\right) \rightarrow\left(13^{+}\right)$ & 2912 & 1811 \\
\hline 121.3 & $70(43)$ & $\left(13^{+}\right) \rightarrow\left(11^{+}\right)$ & 1811 & 1689 \\
644.6 & $76(29)$ & $\left(11^{+}\right) \rightarrow\left(10^{-}\right)$ & 1689 & 1045 \\
\hline \hline
\end{tabular}

known 161, 193, 253, 365, 387, 392, 482, 516, 613, 931, 956, 999, 1096, and $1115 \mathrm{keV}$ transitions are seen. Two new prompt $\gamma$-ray transitions, namely 538 and $1052 \mathrm{keV}$ transitions, are identified and these are marked with an asterisk. The inset shows the tracked Doppler-corrected prompt $\gamma_{P}-\gamma_{P}$ coincidence spectrum with sum gate on the 387, 392, 539 , and $613 \mathrm{keV} \gamma$-ray transitions. This spectrum shows that the $253,365,387,392,482,539,613,931$, and $999 \mathrm{keV} \gamma$ rays are in coincidence. A similar coincidence spectrum is obtained for 161, 193, and $516 \mathrm{keV}$ transitions (not shown in this figure). The delayed singles $\gamma$-ray $\left(\gamma_{D}\right)$ spectrum for $0<t_{\text {decay }}<4 \mu$ s is shown in Fig. 7(b), yielding 85, 120, 131, $144,153,247,852,956$, and $1096 \mathrm{keV}$ transitions, as expected from the previous works. The 85,120 , and $153 \mathrm{keV}$ transitions are newly identified (marked with hash). The inset shows the delayed $\gamma_{D}-\gamma_{D}$ coincidence spectrum with gate on $247 \mathrm{keV}$ transition, yielding 131, 852, and $1096 \mathrm{keV}$ transitions, as reported in previous measurements. The newly observed delayed transitions are placed in accordance with that of ${ }^{125} \mathrm{Sb}$ and summation of gammas. Similarly, Fig. 7(c) shows the singles $\gamma_{D}$-ray spectrum for $10<t_{\text {decay }}<100 \mu$ s, leading to $806,825,1096$, and $1115 \mathrm{keV}$ transitions. The inset shows the delayed $\gamma_{D^{-}} \gamma_{D}$ coincidence spectrum with gate on $1115 \mathrm{keV}$ transition, yielding $806 \mathrm{keV} \gamma$ ray as expected from previous works. The $\gamma$-ray transition, $69 \mathrm{keV}$, between the $\left(19 / 2^{-}\right)$and $15 / 2^{-}$(similar 200 and $141 \mathrm{keV}$ transitions were observed in ${ }^{123,125} \mathrm{Sb}$ isotopes, respectively) could not be observed with the present setup because it is below the energy threshold. Assuming similar structure with the other odd- $A$ Sb isotopes, the $\left(19 / 2^{-}\right)$state can be assumed to be an isomeric state in ${ }^{127} \mathrm{Sb}$. The tracked Doppler-corrected $\gamma_{P}$ in coincidence with the sum gate of $\gamma_{D}$ s; namely, 85, 120, 131, 144, 247, 852,956 , and $1096 \mathrm{keV}$ transitions (for $0<t_{\text {decay }}<4 \mu \mathrm{s}$ ) is shown in Fig. 7(d). This spectrum yields the prompt $\gamma$ ray transitions, $161,253,365,387,392,482,539,613$, and $931 \mathrm{keV}$ transitions. In the previous Ref. [37], these prompt transitions were assigned negative-parities and placed above 


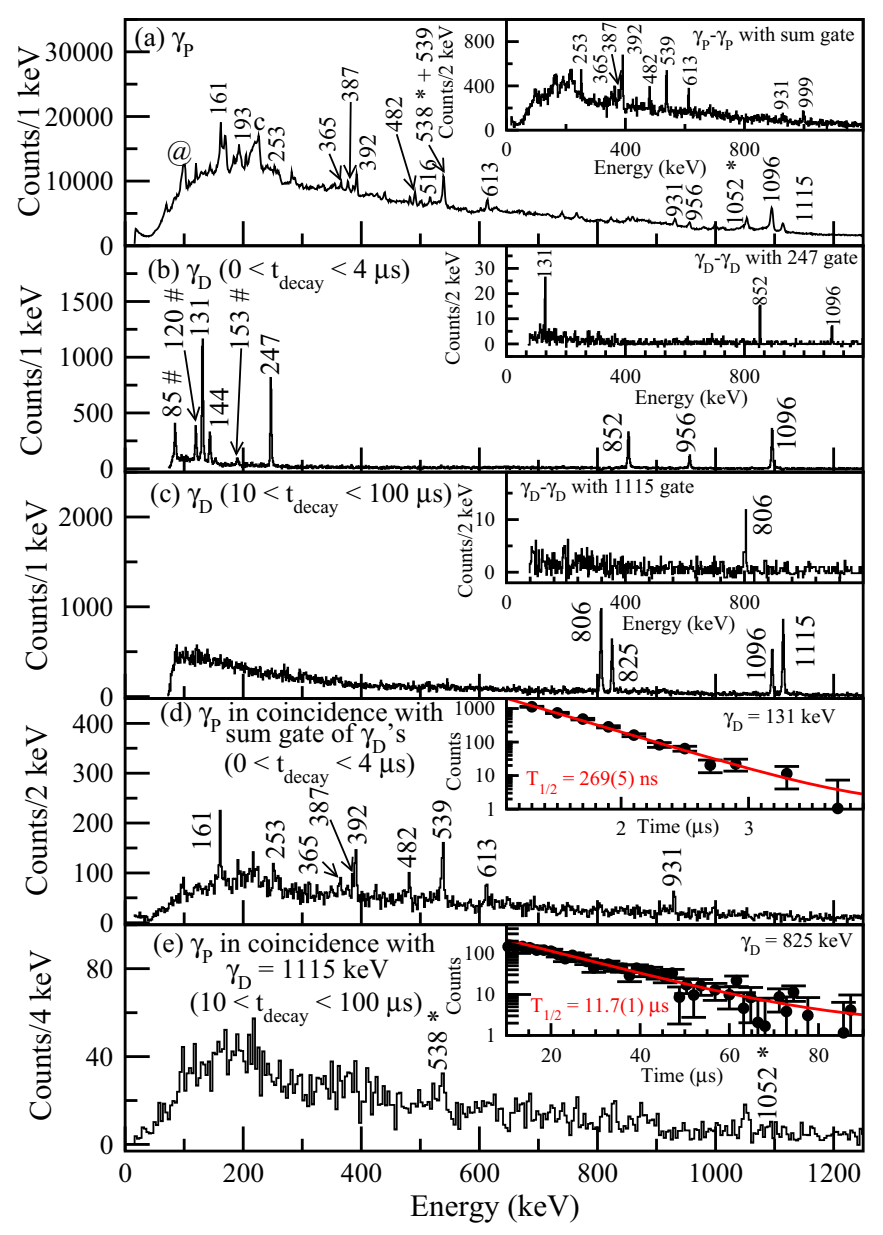

FIG. 7. $A$ - and $Z$-gated $\gamma$-ray spectra for ${ }^{127} \mathrm{Sb}$ : (a) The tracked Doppler-corrected prompt singles $\gamma$-ray $\left(\gamma_{P}\right)$ spectrum with the new $\gamma$-ray transitions marked with asterisk. The inset shows the prompt $\gamma_{P}-\gamma_{P}$ coincidence spectrum with a sum gate on $\gamma_{P}$; namely, 387, 392,539 , and $613 \mathrm{keV} \gamma$ rays. (b), (c) The delayed singles $\gamma$-ray $\left(\gamma_{D}\right)$ spectra for $0<t_{\text {decay }}<4 \mu \mathrm{s}$ and $10<t_{\text {decay }}<100 \mu$ s, respectively. The insets in panels (b) and (c) show the delayed $\gamma_{D}-\gamma_{D}$ coincidence spectra with the gate on 247 and $1115 \mathrm{keV} \gamma$ rays. (d) Tracked Doppler-corrected $\gamma_{P}$ in coincidence with the sum gate of the $\gamma_{D}$; namely, 85, 120, 131, 144, 247, 852, 956, and $1096 \mathrm{keV} \gamma$ rays (for $\left.0<t_{\text {decay }}<4 \mu \mathrm{s}\right)$. The inset shows the decay curve along with the fit for the $131 \mathrm{keV}$ transition. (e) Tracked Doppler-corrected $\gamma_{P}$ in coincidence with the $\gamma_{D}=1115 \mathrm{keV} \gamma$-ray (for $10<t_{\text {decay }}<100 \mu \mathrm{s}$ ). The inset shows the decay curve along with the fit for the $825 \mathrm{keV}$ transition.

the $\left(19 / 2^{-}\right)$isomer. Instead, we suggest these to be positiveparity states (placed above the $23 / 2^{+}$isomer), as observed from the prompt-delayed coincidences. The half-life fit (onecomponent), shown in the inset of Fig. 7(c), for the decay spectrum upon gating on $131 \mathrm{keV}$ transition yields a value of $T_{1 / 2}=269(5) \mathrm{ns}$ for the $23 / 2^{+}$state [close to the value of 234(12) ns reported in Ref. [24]], which has been shown in the inset. This gives $B\left(E 2 ; 23 / 2^{+} \rightarrow 19 / 2^{+}\right)=33.4(7) e^{2} \mathrm{fm}^{4}$. However, the tracked Doppler-corrected $\gamma_{P}$ in coincidence with $\gamma_{D}=1115 \mathrm{keV}$ for $10<t_{\text {decay }}<100 \mu \mathrm{s}$, resulted in the observation of the newly identified 538 and $1052 \mathrm{keV} \gamma$
TABLE VI. Properties of the transitions assigned to ${ }^{127} \mathrm{Sb}$ obtained in this work. The top and bottom panels, separated by a line, are for the prompt and delayed transitions, respectively.

\begin{tabular}{lcccc}
\hline \hline $\mathrm{E}_{\gamma}$ & $\mathrm{I}_{\gamma}$ & $J_{i}^{\pi} \rightarrow J_{f}^{\pi}$ & $E_{i}$ & $E_{f}$ \\
\hline 161.4 & $38(2)$ & $\left(21 / 2^{+}\right) \rightarrow 23 / 2^{+}$ & 2486 & 2325 \\
192.6 & $15(2)$ & $\left(23 / 2^{+}\right) \rightarrow\left(21 / 2^{+}\right)$ & 2678 & 2486 \\
252.7 & $11(5)$ & $\left(37 / 2^{+}\right) \rightarrow\left(35 / 2^{+}\right)$ & 5354 & 5101 \\
365.1 & $12(2)$ & $\left(35 / 2^{+}\right) \rightarrow\left(33 / 2^{+}\right)$ & 5101 & 4736 \\
386.7 & $31(3)$ & $\left(31 / 2^{+}\right) \rightarrow\left(29 / 2^{+}\right)$ & 4255 & 3868 \\
391.8 & $60(3)$ & $\left(27 / 2^{+}\right) \rightarrow\left(25 / 2^{+}\right)$ & 3256 & 2864 \\
482.0 & $17(5)$ & $\left(33 / 2^{+}\right) \rightarrow\left(31 / 2^{+}\right)$ & 4736 & 4255 \\
516.1 & $13(3)$ & $\left(25 / 2^{+}\right) \rightarrow\left(23 / 2^{+}\right)$ & 3194 & 2678 \\
538.1 & $30(2)$ & $\left(27 / 2^{-}\right) \rightarrow\left(23 / 2^{-}\right)$ & 3579 & 3041 \\
539.1 & 100 & $\left(25 / 2^{+}\right) \rightarrow 23 / 2^{+}$ & 2864 & 2325 \\
612.8 & $46(3)$ & $\left(29 / 2^{+}\right) \rightarrow\left(27 / 2^{+}\right)$ & 3868 & 3256 \\
930.9 & $32(2)$ & $\left(27 / 2^{+}\right) \rightarrow\left(23 / 2^{+}\right)$ & 3256 & 2325 \\
999.0 & $21(3)$ & $\left(31 / 2^{+}\right) \rightarrow\left(27 / 2^{+}\right)$ & 4255 & 3256 \\
1052.4 & 100 & $\left(23 / 2^{-}\right) \rightarrow\left(19 / 2^{-}\right)$ & 3041 & 1989 \\
\hline 84.8 & $11(9)$ & $\left(17 / 2^{-}\right) \rightarrow\left(19 / 2^{-}\right)$ & 2074 & 1989 \\
119.9 & $12(7)$ & $19 / 2^{+} \rightarrow\left(17 / 2^{-}\right)$ & 2194 & 2074 \\
131.1 & $49(28)$ & $23 / 2^{+} \rightarrow 19 / 2^{+}$ & 2325 & 2194 \\
143.5 & $14(7)$ & $19 / 2^{+} \rightarrow 15 / 2^{+}$ & 2194 & 2051 \\
153.1 & $2(1)$ & $\left(17 / 2^{-}\right) \rightarrow 15 / 2^{-}$ & 2074 & 1920 \\
246.9 & $47(20)$ & $19 / 2^{+} \rightarrow 15 / 2^{+}$ & 2194 & 1948 \\
806.0 & $97(33)$ & $15 / 2^{-} \rightarrow 9 / 2^{+}$ & 1920 & 1115 \\
824.8 & $61(21)$ & $15 / 2^{-} \rightarrow 11 / 2^{+}$ & 1920 & 1096 \\
852.5 & $43(15)$ & $15 / 2^{+} \rightarrow 11 / 2^{+}$ & 1948 & 1096 \\
956.2 & $17(6)$ & $15 / 2^{+} \rightarrow 11 / 2^{+}$ & 2051 & 1096 \\
1095.8 & $61(20)$ & $11 / 2^{-} \rightarrow 7 / 2^{+}$ & 1096 & 0 \\
1114.6 & 100 & $9 / 2^{+} \rightarrow 7 / 2^{+}$ & 1115 & 0 \\
\hline \hline & & & & \\
\hline \hline
\end{tabular}

rays. These two transitions are thus placed above the $\left(19 / 2^{-}\right)$ isomer, in accordance with the other odd- $A \mathrm{Sb}$ isotopes. The inset shows the half-life fit (two-component with one component fixed at $269 \mathrm{~ns}$ ) for the decay spectrum upon gating on $825 \mathrm{keV}$ transition, which yields a value of $T_{1 / 2}=11.7(1) \mu \mathrm{s}$ for the $15 / 2^{-}$isomer [in agreement with the value of 11(1) $\mu \mathrm{s}$ reported in Ref. [40]].

\section{G. ${ }^{128} \mathrm{Sb}$}

A previous $\gamma$-ray spectroscopy measurement on the highspin states in ${ }^{128} \mathrm{Sb}$ was shown in Ref. [6]. The level scheme as obtained in the present work is shown in Fig. 1. Table VII shows the properties of all the transitions assigned in this work.

The $A$ - and $Z$-gated $\gamma$-ray spectra for ${ }^{128} \mathrm{Sb}$ is shown in Fig. 8. The tracked Doppler-corrected prompt singles $\gamma$-ray spectrum $\left(\gamma_{P}\right)$ for ${ }^{128} \mathrm{Sb}$ is shown in Fig. 8(a). The already known $\gamma$ rays, namely 288, 364, 773, 911, and $1061 \mathrm{keV}$, are seen. In addition, this spectrum shows many new prompt $\gamma$ rays; namely, the $211,245,264,414,497,541,613$, and $1195 \mathrm{keV}$ transitions (marked with an asterisk). Besides, the 128 and $973 \mathrm{keV}$ transitions are also seen (marked with a hash) are observed in both $\gamma_{P}$ [Fig. 8(a)] and delayed spectrum $\left(\gamma_{D}\right)$ [Fig. 8(b)]. The inset in Fig. 8(a) shows the prompt $\gamma-\gamma$ coincidence spectrum $\left(\gamma_{P}-\gamma_{P}\right)$ with a sum gate on the 264 , 364 and $414 \mathrm{keV}$ prompt transitions. This spectrum shows 

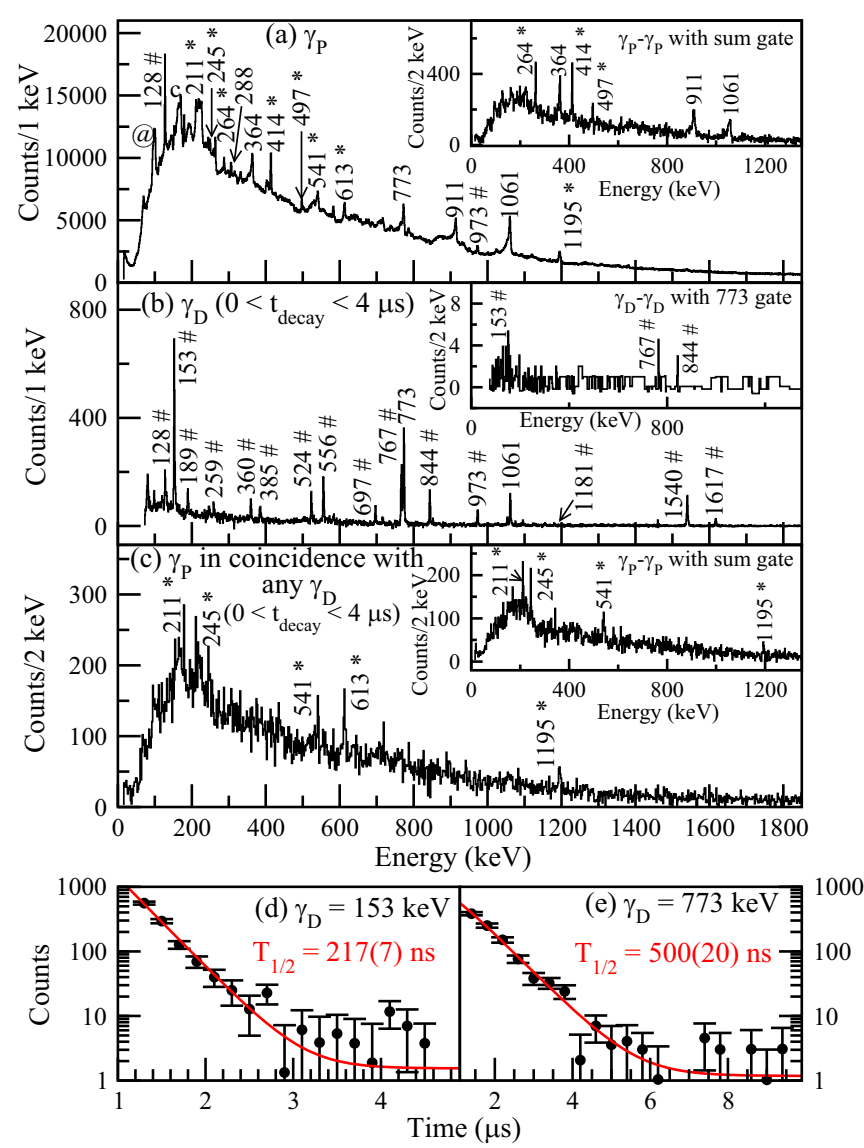

FIG. 8. $A$ - and $Z$-gated $\gamma$-ray spectra for ${ }^{128} \mathrm{Sb}$ : (a) Tracked Doppler-corrected Prompt singles $\gamma$-ray spectrum $\left(\gamma_{P}\right)$. The newly identified prompt and delayed $\gamma$ rays are marked with an asterisk and hash, respectively. The inset shows the $\gamma_{P}-\gamma_{P}$ coincidence spectrum with sum gate on the $\gamma_{P}$ 's, namely the 264, 364 and $414 \mathrm{keV}$ transitions. (b) The delayed singles $\gamma$-ray spectrum $\left(\gamma_{D}\right)$ for $0<t_{\text {decay }}<4 \mu \mathrm{s}$. The newly identified delayed $\gamma$ rays are marked with hash. The inset shows the $\gamma_{D^{-}} \gamma_{D}$ coincidence spectrum with gate on the delayed $773 \mathrm{keV} \gamma$-ray. (c) The $\gamma_{P}$ in coincidence with any delayed $\gamma$-ray for $0<t_{\text {decay }}<4 \mu \mathrm{s}$. Again, the newly identified prompt transitions are marked with an asterisk. The inset shows the $\gamma_{P}-\gamma_{P}$ coincidence spectrum with sum gate on many $\gamma_{P}$ 's, namely the 541 and $1195 \mathrm{keV}$ transitions. Panels (d) and (e) show the decay curves along with the fits for the delayed 153 and $773 \mathrm{keV}$ transitions, respectively.

that the 264, 364, 414, 497, 911, and $1061 \mathrm{keV}$ transitions are in coincidence. The delayed $\gamma$-ray $\left(\gamma_{D}\right)$ singles spectrum, with $0<t_{\text {decay }}<4 \mu$ s is shown in Fig. 8(b). This spectrum shows new delayed $\gamma$-ray transitions; namely, 128, 153, 189, $259,360,385,524,556,697,767,844,973,1181,1540$, and $1617 \mathrm{keV}$ (marked with hash), in addition to the known 773 and $1061 \mathrm{keV}$ transition. The delayed $\gamma-\gamma$ coincidence spectrum $\left(\gamma_{D}-\gamma_{D}\right)$ with gate on the $773 \mathrm{keV}$ transition is shown in the inset of Fig. 8(b). This spectrum shows that the 153, 767, and $844 \mathrm{keV}$ are in coincidence with the $773 \mathrm{keV}$ transition. Figure $8(\mathrm{c})$ shows the prompt spectrum $\left(\gamma_{P}\right)$ in coincidence with any delayed $\gamma$-ray transition with $0<t_{\text {decay }}<4 \mu \mathrm{s}$ is shown in Fig. 8(c). The inset in Fig. 8(c) shows the $\gamma_{P}-\gamma_{P}$
TABLE VII. Properties of the transitions assigned to ${ }^{128} \mathrm{Sb}$ obtained in this work. The top and bottom panels, separated by a line, are for the prompt and delayed transitions, respectively.

\begin{tabular}{lcrrr}
\hline \hline $\mathrm{E}_{\gamma}$ & $\mathrm{I}_{\gamma}$ & $J_{i}^{\pi} \rightarrow J_{f}^{\pi}$ & $E_{i}$ & $E_{f}$ \\
\hline 211.1 & $10(5)$ & $\left(18^{+}\right) \rightarrow\left(17^{+}\right)$ & 3961 & 3749 \\
244.8 & $20(4)$ & $\left(17^{+}\right) \rightarrow\left(16^{+}\right)$ & 3749 & 3505 \\
263.9 & $37(4)$ & $\left(16^{-}\right) \rightarrow\left(15^{-}\right)$ & 3011 & 2748 \\
288.4 & $27(3)$ & $\left(10^{-}\right) \rightarrow\left(9^{-}\right)$ & 1061 & 773 \\
364.3 & $60(1)$ & $\left(14^{-}\right) \rightarrow\left(12^{-}\right)$ & 2333 & 1969 \\
413.9 & $52(2)$ & $\left(15^{-}\right) \rightarrow\left(14^{-}\right)$ & 2748 & 2333 \\
497.0 & $20(1)$ & $\left(17^{-}\right) \rightarrow\left(16^{-}\right)$ & 3508 & 3011 \\
541.3 & $58(3)$ & $\left(16^{+}\right) \rightarrow\left(15^{+}\right)$ & 3505 & 2964 \\
612.9 & 100 & $\left(12^{+}\right) \rightarrow\left(11^{+}\right)$ & 2230 & 1617 \\
773.0 & 100 & $\left(9^{-}\right) \rightarrow\left(8^{-}\right)$ & 773 & 0 \\
910.9 & $76(2)$ & $\left(12^{-}\right) \rightarrow\left(10^{-}\right)$ & 1969 & 1061 \\
1061.4 & $46(2)$ & $\left(10^{-}\right) \rightarrow\left(8^{-}\right)$ & 1061 & 0 \\
1195.1 & 100 & $\left(15^{+}\right) \rightarrow\left(13^{+}\right)$ & 2964 & 1769 \\
\hline 127.8 & $12(7)$ & $\left(9^{+}\right) \rightarrow\left(8^{+}\right)$ & 1540 & 1412 \\
152.6 & $45(24)$ & $\left(13^{+}\right) \rightarrow\left(11^{+}\right)$ & 1769 & 1617 \\
189.4 & $11(5)$ & $\left(8^{+}\right) \rightarrow\left(7^{+}\right)$ & 1412 & 1223 \\
259.4 & $11(5)$ & $\left(7^{-}\right) \rightarrow\left(8^{-}\right)$ & 259 & 0 \\
359.9 & $14(6)$ & $\left(7^{+}\right) \rightarrow\left(8^{-}\right)$ & 360 & 0 \\
384.8 & $13(5)$ & $\left(11^{+}\right) \rightarrow\left(9^{-}\right)$ & 1617 & 1232 \\
523.6 & $25(9)$ & $\left(7^{+}\right) \rightarrow\left(6^{+}\right)$ & 1223 & 697 \\
556.2 & $42(15)$ & $\left(11^{+}\right) \rightarrow\left(10^{-}\right)$ & 1617 & 1061 \\
696.9 & $17(6)$ & $\left(6^{+}\right) \rightarrow\left(8^{-}\right)$ & 697 & 0 \\
767.0 & $63(22)$ & $\left(9^{+}\right) \rightarrow\left(9^{-}\right)$ & 1540 & 773 \\
843.8 & $32(11)$ & $\left(11^{+}\right) \rightarrow\left(9^{-}\right)$ & 1617 & 773 \\
973.3 & $20(7)$ & $\left(9^{-}\right) \rightarrow\left(7^{-}\right)$ & 1232 & 259 \\
1181.2 & $4(2)$ & $\left(9^{+}\right) \rightarrow\left(7^{-}\right)$ & 1540 & 360 \\
1540.4 & $43(15)$ & $\left(9^{+}\right) \rightarrow\left(8^{-}\right)$ & 1540 & 0 \\
1617.2 & $10(3)$ & $\left(11^{+}\right) \rightarrow\left(8^{-}\right)$ & 1617 & 0 \\
\hline \hline & & & & $\rightarrow$ \\
\hline \hline
\end{tabular}

coincidence spectrum with gate on the newly identified 541 and $1195 \mathrm{keV}$ transitions. This spectrum shows that 211, 245,541 , and $1195 \mathrm{keV}$ transitions are in coincidence and placed above the $\left(13^{+}\right)$isomer. But Fig. 8(c) shows $613 \mathrm{keV}$ transition which is not present in the inset of Fig. 8(c). Hence, the $613 \mathrm{keV}$ transition is placed above the $\left(11^{+}\right)$ isomer. Figures 8(d) and 8(e) show the decay curves for 153 and $773 \mathrm{keV}$ transitions, respectively. An exponential fit for these curves yields values 217(7) ns (using a onecomponent fit) and 500(20) ns (using a two-component fit with one component fixed at $217 \mathrm{~ns}$ ), respectively. This gives $B\left(E 2 ; 13^{+} \rightarrow 11^{+}\right)=23.1(8) e^{2} \mathrm{fm}^{4}$.

\section{H. ${ }^{129} \mathrm{Sb}$}

References [25,41,42] reported the previous $\gamma$-ray spectroscopy measurements on ${ }^{129} \mathrm{Sb}$. The level scheme as obtained in the present work is shown in Fig. 1. Table VIII shows the properties of all the transitions assigned in this work.

The $A$ - and $Z$-gated $\gamma$-ray spectra for ${ }^{129} \mathrm{Sb}$ are shown in Fig. 9. The tracked Doppler-corrected prompt singles $\gamma$-ray spectrum $\left(\gamma_{P}\right)$ for ${ }^{129} \mathrm{Sb}$ is shown in Fig. 9(a). The previously observed 1128 and $1161 \mathrm{keV}$ transitions are seen. Many new prompt $\gamma$-ray transitions; namely, 156, 315, 471, 530, 622, 


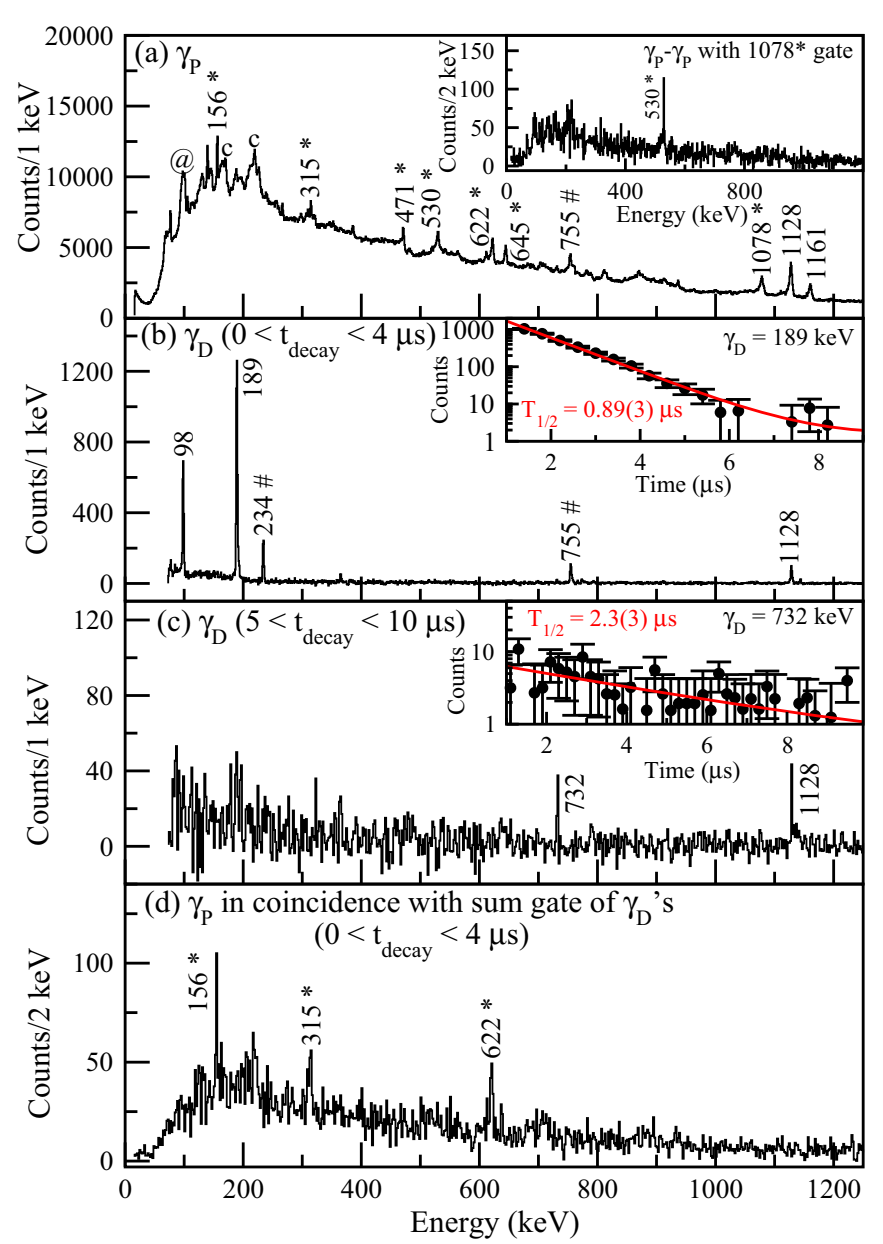

FIG. 9. $A$ - and $Z$-gated $\gamma$-ray spectra for ${ }^{129} \mathrm{Sb}$ : (a) The tracked Doppler-corrected prompt singles $\gamma$-ray $\left(\gamma_{P}\right)$ spectrum with the new $\gamma$-ray transitions marked with an asterisk. The inset shows the tracked Doppler-corrected prompt $\gamma_{P}-\gamma_{P}$ coincidence spectrum with gate on the newly identified $1078 \mathrm{keV}$ transition. (b), (c) The delayed singles $\gamma$-ray $\left(\gamma_{D}\right)$ spectra for $0<t_{\text {decay }}<4 \mu$ s and $5<t_{\text {decay }}<10 \mu \mathrm{s}$, respectively. The insets in panels (b) and (c) show the decay curves along with the fits for the 189 and $732 \mathrm{keV}$ transitions, respectively. (d) Tracked Doppler-corrected $\gamma_{P}$ in coincidence with the sum gate of the $\gamma_{D} \mathrm{~s}$; namely, 98, 189, 234, and $755 \mathrm{keV}$ delayed transitions (for $0<t_{\text {decay }}<4 \mu \mathrm{s}$ ).

645, and $1078 \mathrm{keV}$ transitions are identified (marked with an asterisk). The $755 \mathrm{keV}$ (marked with a hash) is observed in both prompt $\left(\gamma_{P}\right)$ [Fig. 9(a)] and delayed spectrum $\left(\gamma_{D}\right)$ [Fig. 9(b)]. The $1161 \mathrm{keV}$ is actually depopulating the $9 / 2^{+}$ state to the ground state $[41,42]$, and this is not shown in the current level scheme. The inset shows the tracked Doppler-corrected prompt $\gamma_{P}-\gamma_{P}$ coincidence spectrum with gate on the newly identified $1078 \mathrm{keV}$ transition. This spectrum shows that the 530 and $1078 \mathrm{keV}$ transitions are in coincidence. A similar coincidence spectrum is obtained for the 315 and $622 \mathrm{keV}$ transitions (not shown in this figure). However, no coincidences are seen upon gating on the 471 and $645 \mathrm{keV}$ transitions, and hence these are not placed in the level scheme. The delayed singles $\gamma$-ray $\left(\gamma_{D}\right)$ spectrum for $0<t_{\text {decay }}<4 \mu \mathrm{s}$ is shown in Fig. 9(b), yielding 98, 189,
TABLE VIII. Properties of the transitions assigned to ${ }^{129} \mathrm{Sb}$ obtained in this work. The top and bottom panels, separated by a line, are for the prompt and delayed transitions, respectively.

\begin{tabular}{lcccc}
\hline \hline $\mathrm{E}_{\gamma}$ & $\mathrm{I}_{\gamma}$ & $J_{i}^{\pi} \rightarrow J_{f}^{\pi}$ & $E_{i}$ & $E_{f}$ \\
\hline 156.1 & $55(4)$ & $\left(21 / 2^{+}\right) \rightarrow 23 / 2^{+}$ & 2295 & 2139 \\
314.7 & $44(4)$ & $\left(27 / 2^{+}\right) \rightarrow\left(25 / 2^{+}\right)$ & 3075 & 2761 \\
529.7 & $69(10)$ & $\left(27 / 2^{-}\right) \rightarrow\left(23 / 2^{-}\right)$ & 3469 & 2939 \\
622.1 & 100 & $\left(25 / 2^{+}\right) \rightarrow 23 / 2^{+}$ & 2761 & 2139 \\
1078.6 & 100 & $\left(23 / 2^{-}\right) \rightarrow 19 / 2^{-}$ & 2939 & 1851 \\
\hline 98.3 & $38(7)$ & $23 / 2^{+} \rightarrow 19 / 2^{+}$ & 2139 & 2040 \\
189.2 & $71(34)$ & $19 / 2^{+} \rightarrow 19 / 2^{-}$ & 2040 & 1851 \\
234.0 & $35(16)$ & $19 / 2^{+} \rightarrow\left(17 / 2^{-}\right)$ & 2040 & 1806 \\
732.5 & $47(17)$ & $15 / 2^{-} \rightarrow 11 / 2^{+}$ & 1861 & 1128 \\
755.0 & $37(15)$ & $\left(15 / 2^{+}\right) \rightarrow 11 / 2^{+}$ & 1883 & 1128 \\
1128.5 & 100 & $11 / 2^{+} \rightarrow 7 / 2^{+}$ & 1128 & 0 \\
\hline \hline
\end{tabular}

234, 755, and $1128 \mathrm{keV}$ transitions, as expected from the previous works. The 234 and $755 \mathrm{keV}$ transitions are newly observed (marked with a hash). The $234 \mathrm{keV}$ is placed below the $19 / 2^{+}$state decaying to $\left(17 / 2^{-}\right)$state, by following the systematics with the $120 \mathrm{keV}$ in ${ }^{127} \mathrm{Sb}$ and $108 \mathrm{keV}$ in ${ }^{131} \mathrm{Sb}$. The $755 \mathrm{keV}$ on the other hand is assigned to be depopulating the $\left(15 / 2^{+}\right)$to the $11 / 2^{+}$state, in accordance with the $852 \mathrm{keV}$ in ${ }^{127} \mathrm{Sb}$ and the shell-model calculations. The required $157 \mathrm{keV}$ from $19 / 2^{+}$to $\left(15 / 2^{+}\right)$, as seen in the lower odd- $A \mathrm{Sb}$ isotopes $\left(442,332,247 \mathrm{keV}\right.$ in ${ }^{123-127} \mathrm{Sb}$, respectively), is not seen in the delayed spectrum. The halflife fit (one-component) for the decay spectrum upon gating on $189 \mathrm{keV}$ transition yields a value of $T_{1 / 2}=0.89(3) \mu \mathrm{s}$ [in agreement with the value of 1.1(1) $\mu$ s reported in Ref. [25]], which gives $B\left(E 2 ; 23 / 2^{+} \rightarrow 19 / 2^{+}\right)=25.3(8) e^{2} \mathrm{fm}^{4}$ for the $23 / 2^{+}$state. Similarly, Fig. 9(c) shows the $\gamma_{D}$-ray spectrum for $5<t_{\text {decay }}<10 \mu \mathrm{s}$, leading to 732 and $1128 \mathrm{keV}$ transitions. A half-life fit (two-component with one component fixed to $0.89 \mu \mathrm{s}$ ) for the decay spectrum upon gating on $732 \mathrm{keV}$ transition yields a value of $T_{1 / 2}=2.3(3) \mu \mathrm{s}$ for the $15 / 2^{-}$state [in agreement with the value of 2.2(2) $\mu \mathrm{s}$ reported in Ref. [25]]. The tracked Doppler-corrected $\gamma_{P}$ in coincidence with the sum gate of $\gamma_{D}$ s; namely, 98, 189, 234 , and $755 \mathrm{keV}$ delayed transitions $\left(0<t_{\text {decay }}<4 \mu \mathrm{s}\right)$ is shown in Fig. 9(d). This spectrum yields the newly identified prompt 156, 315, and $622 \mathrm{keV} \gamma$-ray transitions. Thus, all these transitions are placed above the $23 / 2^{+}$isomer. Since the $156 \mathrm{keV}$ transition is not observed in coincidence with 315 and $622 \mathrm{keV}$ transitions (not shown in this figure), it is assigned depopulating from $\left(21 / 2^{+}\right)$to $23 / 2^{+}$state, in accordance with the $161 \mathrm{keV}$ in ${ }^{127} \mathrm{Sb}$ and $206 \mathrm{keV}$ in ${ }^{131} \mathrm{Sb}$. In addition, the $\gamma_{D}$ in coincidence with all these newly observed prompt transitions are studied and all of these yield the 98, 189 , and $1128 \mathrm{keV}$ transitions (not shown in this figure). The tracked Doppler-corrected $\gamma_{P}$ in coincidence with any $\gamma_{D}$ for $5<t_{\text {decay }}<10 \mu \mathrm{s}$ did not result in any new prompt $\gamma$ rays and hence no transitions are placed above the $15 / 2^{-}$ isomer. No prompt-delayed correlations could be carried out for the $19 / 2^{-}$isomer because it has a very long half-life of $17.7 \mathrm{~min}$. As the inset of Fig. 9(a) shows the coincidence 


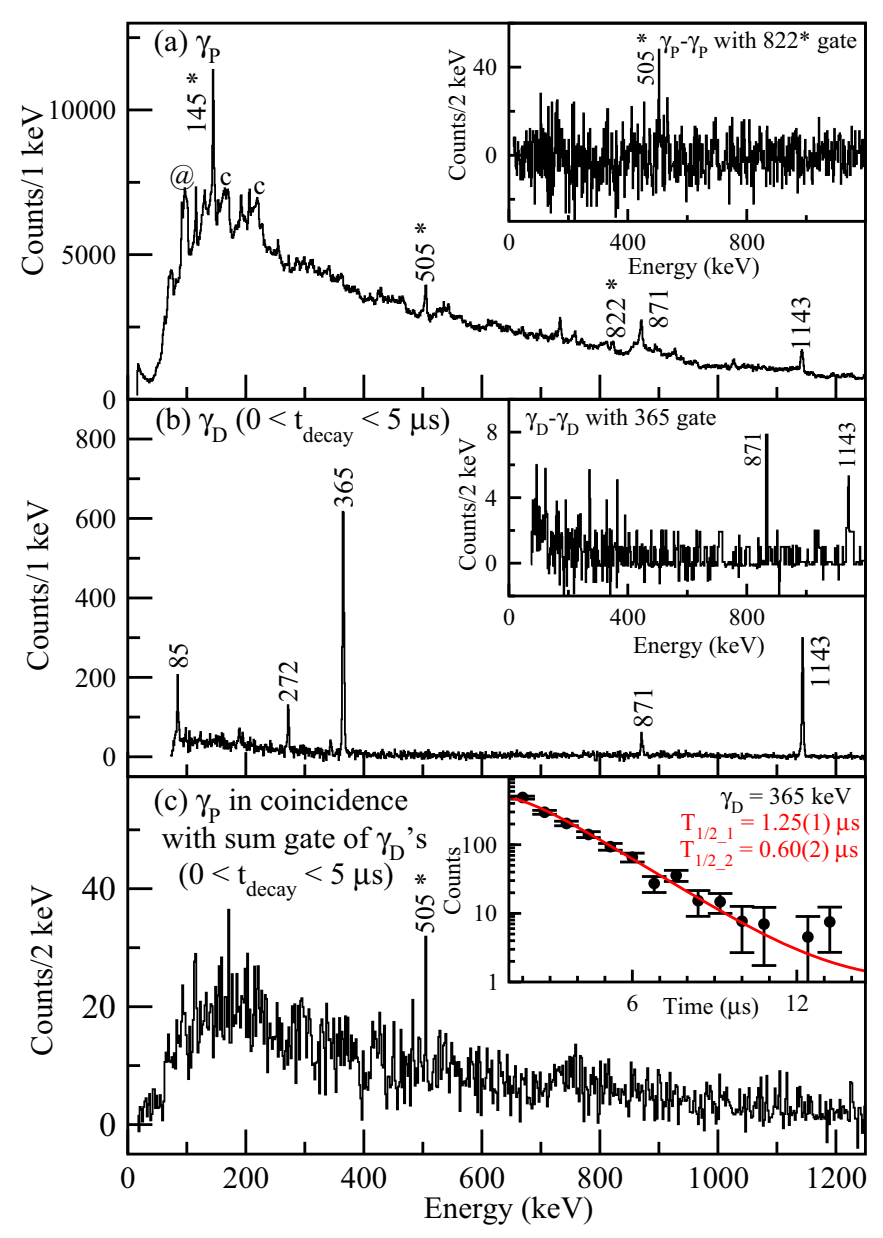

FIG. 10. $A$ - and $Z$-gated $\gamma$-ray spectra for ${ }^{130} \mathrm{Sb}$ : (a) Tracked Doppler-corrected prompt singles $\gamma$-ray spectrum $\left(\gamma_{P}\right)$. The newly identified prompt $\gamma$ rays are marked with an asterisk. The inset shows the tracked Doppler-corrected $\gamma_{P}-\gamma_{P}$ coincidence spectrum with gate on the newly identified $822 \mathrm{keV}$ prompt $\gamma$-ray. (b) The delayed singles $\gamma$-ray spectrum $\left(\gamma_{D}\right)$ for $0<t_{\text {decay }}<5 \mu$ s. The inset shows the $\gamma_{D}-\gamma_{D}$ coincidence spectrum with gate on the delayed $365 \mathrm{keV} \gamma$ ray. (c) The tracked Doppler-corrected $\gamma_{P}$ in coincidence with the delayed $\gamma$ rays $=365$ and $1143 \mathrm{keV}$ transitions, for $0<t_{\text {decay }}<5 \mu \mathrm{s}$. The newly identified prompt transitions are marked with an asterisk. The inset shows the decay curve along with the two-component fit for the delayed $365 \mathrm{keV}$ transition.

of 530 and $1078 \mathrm{keV}$ transitions and a similar systematics has been observed in the lower odd- $A$ Sb isotopes, these transitions are placed above the $19 / 2^{-}$isomer.

\section{I. ${ }^{130} \mathrm{Sb}$}

A previous measurement on the high-spin states using $\gamma$ ray spectroscopy in ${ }^{130} \mathrm{Sb}$ was reported in Ref. [43]. The level scheme as obtained in the present work is shown in Fig. 1. Table IX shows the properties of all the transitions assigned in this work.

The $A$ - and $Z$-gated $\gamma$-ray spectra for ${ }^{130} \mathrm{Sb}$ are shown in Fig. 10. The tracked Doppler-corrected prompt singles $\gamma$-ray spectrum $\left(\gamma_{P}\right)$ for ${ }^{130} \mathrm{Sb}$ is shown in Fig. 10(a). The already known 871 and $1143 \mathrm{keV} \gamma$ rays are observed. In addition,
TABLE IX. Properties of the transitions assigned to ${ }^{130} \mathrm{Sb}$ obtained in this work. The top and bottom panels, separated by a line, are for the prompt and delayed transitions, respectively.

\begin{tabular}{lccrr}
\hline \hline $\mathrm{E}_{\gamma}$ & $\mathrm{I}_{\gamma}$ & $J_{i}^{\pi} \rightarrow J_{f}^{\pi}$ & $E_{i}$ & $E_{f}$ \\
\hline 504.9 & 100 & $\left(12^{+}\right) \rightarrow\left(11^{+}\right)$ & 2050 & 1545 \\
821.8 & $48(8)$ & $\left(13^{+}\right) \rightarrow\left(12^{+}\right)$ & 2872 & 2050 \\
\hline 84.7 & $11(10)$ & $6^{-} \rightarrow 8^{-}$ & 85 & 0 \\
272.1 & $23(9)$ & $\left(10^{-}\right) \rightarrow\left(9^{-}\right)$ & 1143 & 871 \\
365.2 & $113(42)$ & $\left(11^{+}\right) \rightarrow\left(10^{-}\right)$ & 1508 & 1143 \\
871.1 & $29(7)$ & $\left(9^{-}\right) \rightarrow 8^{-}$ & 871 & 0 \\
1143.4 & 100 & $\left(10^{-}\right) \rightarrow 8^{-}$ & 1143 & 0 \\
\hline \hline
\end{tabular}

three new prompt transitions; namely, 145, 505, and $822 \mathrm{keV}$ transitions are observed, which are marked with an asterisk. The inset in Fig. 10(a) shows the tracked Doppler-corrected prompt $\gamma-\gamma$ coincidence spectrum $\left(\gamma_{P}-\gamma_{P}\right)$ with gate on the newly identified $822 \mathrm{keV}$ transition. This shows that the 505 and $822 \mathrm{keV}$ transitions are in coincidence, as shown in the level scheme. But $145 \mathrm{keV}$ is not observed in coincidence and hence not placed in the level scheme. Figure 10(b) shows the delayed $\gamma$-ray $\left(\gamma_{D}\right)$ singles spectrum with $0<t_{\text {decay }}<5 \mu$ s. All the known delayed $\gamma$ rays; namely, 85, 272, 365, 871, and $1143 \mathrm{keV}$ transitions are observed. The delayed $\gamma-\gamma$ $\left(\gamma_{D}-\gamma_{D}\right)$ coincidence spectrum with gate on $365 \mathrm{keV}$ transition is shown in the inset of Fig. 10(b). Figure 10(c) shows the tracked Doppler-corrected $\gamma_{P}$ in coincidence with the sum gate of $\gamma_{D}=365$ and $1143 \mathrm{keV}$ transitions. This yields the newly identified $505 \mathrm{keV}$ transition. As from the inset of Fig. 10(a), the 505 and $822 \mathrm{keV}$ transitions are seen in coincidence, these two transitions are placed above the $\left(11^{+}\right)$ state, following the systematics with the lower even- $A \mathrm{Sb}$ isotopes. The inset in Fig. 10(c) shows the decay curve for the delayed $365 \mathrm{keV}$ transition. A one-component fit to this transition yielded a value shorter than $1.8(2) \mu \mathrm{s}$ for the $\left(13^{+}\right)$ state [43]. The discrepancy in the $T_{1 / 2}$ of $\left(13^{+}\right)$state may be due to the time of flight of $\sim 2 \mu$ s for the setup used in Ref. [43], that bias the measurement of the half-life to higher values. Thus, the $\left(11^{+}\right)$state might also have a halflife, as observed in the lower even- $A$ Sb isotopes. A two component fit [as shown in the lower inset in Fig. 10(c)] was carried out, which yielded a half-life of $1.25(1) \mu \mathrm{s}$ for the $\left(13^{+}\right)$state and $0.600(15) \mu$ s for the $\left(11^{+}\right)$state. The $B\left(E 2 ; 13^{+} \rightarrow 11^{+}\right)=105(6) e^{2} \mathrm{fm}^{4}$ was obtained.

\section{J. ${ }^{131} \mathrm{Sb}$}

The high-spin $\gamma$-ray spectroscopy of ${ }^{131} \mathrm{Sb}$ was previously reported in Refs. [26,44]. The level scheme as obtained in the present work is shown in Fig. 1. Table $\mathrm{X}$ shows the properties of all the transitions assigned in this work.

The $A$ - and $Z$-gated $\gamma$-ray spectra for ${ }^{131} \mathrm{Sb}$ are shown in Fig. 11. The tracked Doppler-corrected prompt singles $\gamma$-ray spectrum $\left(\gamma_{P}\right)$ for ${ }^{131} \mathrm{Sb}$ is shown in Fig. 11(a). The previously known $1226 \mathrm{keV}$ transition is seen in this spectrum. However the other transitions; namely, 96, 344, 382, and $450 \mathrm{keV}$ are not seen in Fig. 11(a) because these decay directly from long-lived isomers. In addition, many 


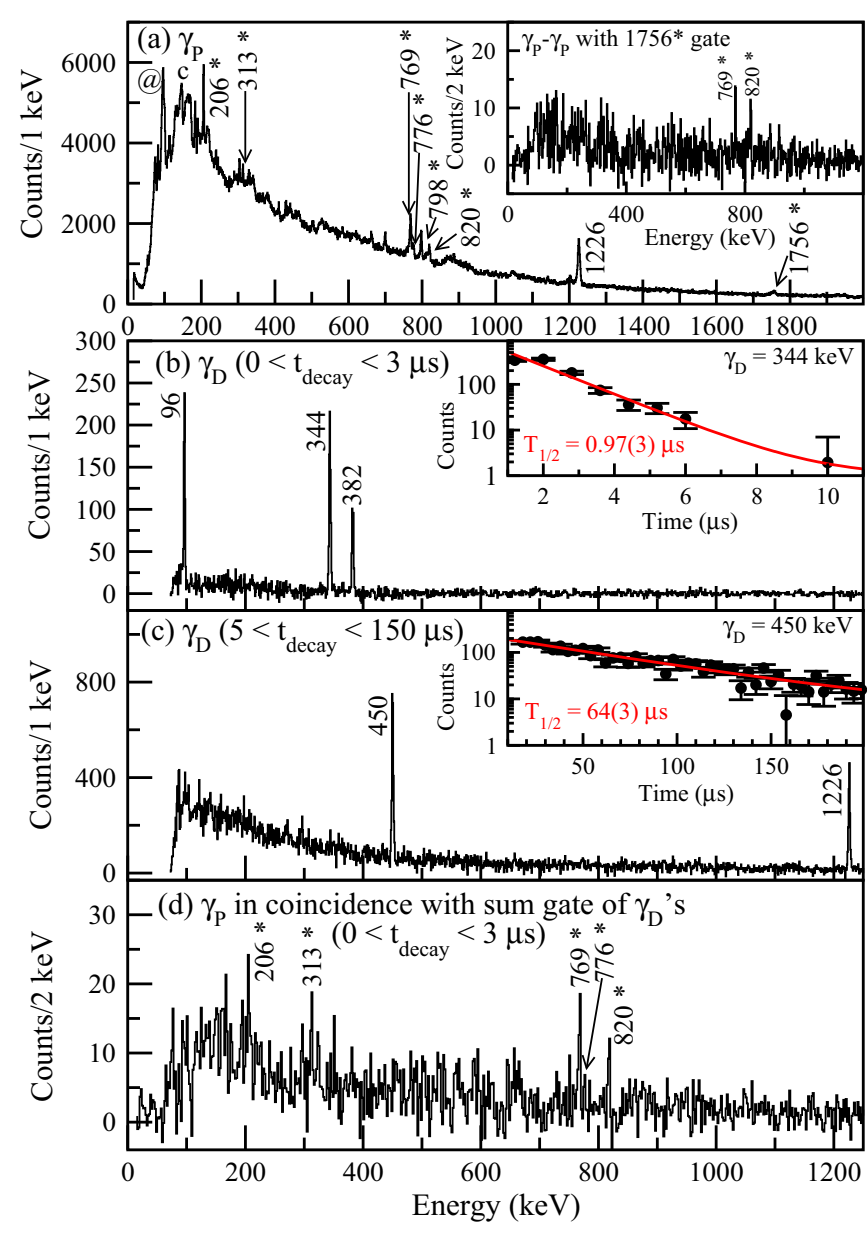

FIG. 11. $A$ - and $Z$-gated $\gamma$-ray spectra for ${ }^{131} \mathrm{Sb}$ : The tracked Doppler-corrected prompt singles $\gamma$-ray $\left(\gamma_{P}\right)$ spectrum with the new $\gamma$-ray transitions marked with asterisk. The inset shows the tracked Doppler-corrected prompt $\gamma_{P}-\gamma_{P}$ coincidence spectrum with gate on the newly identified $1756 \mathrm{keV}$ transition. (b), (c) The delayed singles $\gamma$-ray $\left(\gamma_{D}\right)$ spectra for $0<t_{\text {decay }}<3 \mu$ s and $5<t_{\text {decay }}<150 \mu \mathrm{s}$, respectively. The insets in panels (b) and (c) show the decay curves along with the fits for the 344 and $450 \mathrm{keV}$ transitions, respectively. (d) Tracked Doppler-corrected $\gamma_{P}$ in coincidence with the sum gate of the $\gamma_{D}$; namely, 96, 344, and $382 \mathrm{keV}$ delayed transitions (for $\left.0<t_{\text {decay }}<3 \mu \mathrm{s}\right)$.

new prompt $\gamma$-ray transitions, namely $206,313,769,776$, 798, 820, and $1756 \mathrm{keV}$ transitions, are identified (marked with an asterisk). The inset shows the tracked Dopplercorrected prompt $\gamma_{P}-\gamma_{P}$ coincidence spectrum with gate on the newly identified $1756 \mathrm{keV}$ transition. This spectrum shows that the 769,820 , and $1756 \mathrm{keV}$ transitions are in coincidence. A similar coincidence spectrum is obtained for the 206, 313, and $776 \mathrm{keV}$ transitions (not shown in this figure). However, no coincidences could be observed with a gate on the $798 \mathrm{keV}$ transition, and hence this is not placed in the level scheme. The delayed singles $\gamma$-ray $\left(\gamma_{D}\right)$ spectrum for $0<t_{\text {decay }}<3 \mu \mathrm{s}$ is shown in Fig. 11(b), yielding 96, 344, and $382 \mathrm{keV}$ transitions, as observed in previous measurements. The half-life fit (one-component) for the decay spectrum upon gating on $344 \mathrm{keV}$ transition
TABLE X. Properties of the transitions assigned to ${ }^{131} \mathrm{Sb}$ obtained in this work. The top and bottom panels, separated by a line, are for the prompt and delayed transitions, respectively.

\begin{tabular}{lclcc}
\hline \hline $\mathrm{E}_{\gamma}$ & $\mathrm{I}_{\gamma}$ & \multicolumn{1}{c}{$J_{i}^{\pi} \rightarrow J_{f}^{\pi}$} & $E_{i}$ & $E_{f}$ \\
\hline 206.1 & $56(4)$ & $\left(21 / 2^{+}\right) \rightarrow 23 / 2^{+}$ & 2372 & 2166 \\
313.3 & $16(3)$ & $\left(25 / 2^{+}\right) \rightarrow\left(23 / 2^{+}\right)$ & 3461 & 3148 \\
768.7 & 100 & $\left(25 / 2^{+}\right) \rightarrow 23 / 2^{+}$ & 2935 & 2166 \\
776.0 & $38(5)$ & $\left(23 / 2^{+}\right) \rightarrow\left(21 / 2^{+}\right)$ & 3148 & 2372 \\
820.0 & $43(4)$ & $\left(27 / 2^{+}\right) \rightarrow\left(25 / 2^{+}\right)$ & 3755 & 2935 \\
1755.7 & $36(5)$ & $\left(31 / 2^{+}\right) \rightarrow\left(27 / 2^{+}\right)$ & 5510 & 3755 \\
\hline 96.3 & $11(8)$ & $23 / 2^{+} \rightarrow 19 / 2^{+}$ & 2166 & 2070 \\
343.6 & $30(11)$ & $19 / 2^{+} \rightarrow 17 / 2^{-}$ & 2070 & 1726 \\
382.4 & $16(6)$ & $19 / 2^{+} \rightarrow 19 / 2^{-}$ & 2070 & 1688 \\
450.0 & $61(22)$ & $15 / 2^{-} \rightarrow 11 / 2^{+}$ & 1676 & 1226 \\
1226.2 & 100 & $11 / 2^{+} \rightarrow 7 / 2^{+}$ & 1226 & 0 \\
\hline \hline
\end{tabular}

yields a value of $T_{1 / 2}=0.97(3) \mu$ s for the $23 / 2^{+}$state [in agreement with the value of 1.1(2) $\mu$ s given in Ref. [26]], yielding $B\left(E 2 ; 23 / 2^{+} \rightarrow 19 / 2^{+}\right)=24.6(8) e^{2} \mathrm{fm}^{4}$. Similarly, Fig. 11(c) shows the delayed $\gamma$-ray spectrum for $5<t_{\text {decay }}<150 \mu \mathrm{s}$, leading to 450 and $1226 \mathrm{keV}$ transitions. A half-life fit (using a three component fit with two components fixed to $0.97 \mu \mathrm{s}$ and $4.3 \mu \mathrm{s}$ ) for the decay spectrum upon gating on $450 \mathrm{keV}$ transition yields a value of $T_{1 / 2}=64(3) \mu \mathrm{s}$ for the $15 / 2^{-}$state [in agreement with $65(5) \mu$ s quoted by Ref. [26]]. The $B\left(E 2 ; 19 / 2^{-} \rightarrow 15 / 2^{-}\right)=41(8) e^{2} \mathrm{fm}^{4}$, as given in Ref. [22]. The tracked Doppler-corrected $\gamma_{P}$ in coincidence with the sum gate of the $\gamma_{D}$ s; namely, 96, 344, and $382 \mathrm{keV}$ delayed transitions (for $0<t_{\text {decay }}<3 \mu \mathrm{s}$ ) is shown in Fig. 11(d). This spectrum yields almost all the newly identified prompt $\gamma$-ray transitions, that are placed above the $23 / 2^{+}$isomer. In addition, the $\gamma_{D}$ in coincidence with all the newly observed prompt transitions are studied and all of these yield the 96, 344, and $382 \mathrm{keV}$ transitions (not shown in this figure). However, the tracked Doppler-corrected $\gamma_{P}$ in coincidence with any $\gamma_{D}$ for $5<t_{\text {decay }}<150 \mu \mathrm{s}$, did not result in any new prompt $\gamma$ rays and hence no transitions are placed above the $15 / 2^{-}$isomer. Also, no transitions could be placed above the $19 / 2^{-}$isomer.

\section{DISCUSSION}

A systematic study of both odd- $A$ and even- $A{ }^{122-131} \mathrm{Sb}$ isotopes was carried out in this work. The observed excited states in ${ }^{122-131} \mathrm{Sb}(Z=51)$ isotopes have a close correspondence with those in ${ }^{121-130} \mathrm{Sn}(Z=50)$ isotopes. This can be evinced from the similarities in the energy differences of the low-lying states in the even- $A$ /odd- $A$ Sn i.e., $E\left(2^{+}\right)-E\left(0^{+}\right) \sim E\left(15 / 2^{-}\right)-E\left(11 / 2^{-}\right)$and odd- $A$ /even- $A$ $\mathrm{Sb}$ i.e., $E\left(11 / 2^{+}\right)-E\left(7 / 2^{+}\right) \sim E\left(10^{-}\right)-E\left(8^{-}\right)$, respectively. This is depicted in Fig. 12. Similar correspondence for selected high-spin states is presented in Fig. 13. These similarities are due to the fact that the $\mathrm{Sb}$ isotopes have a single valence proton particle in the $g_{7 / 2}$ orbital in addition to neutrons in the corresponding $\mathrm{Sn}$ isotopes. 


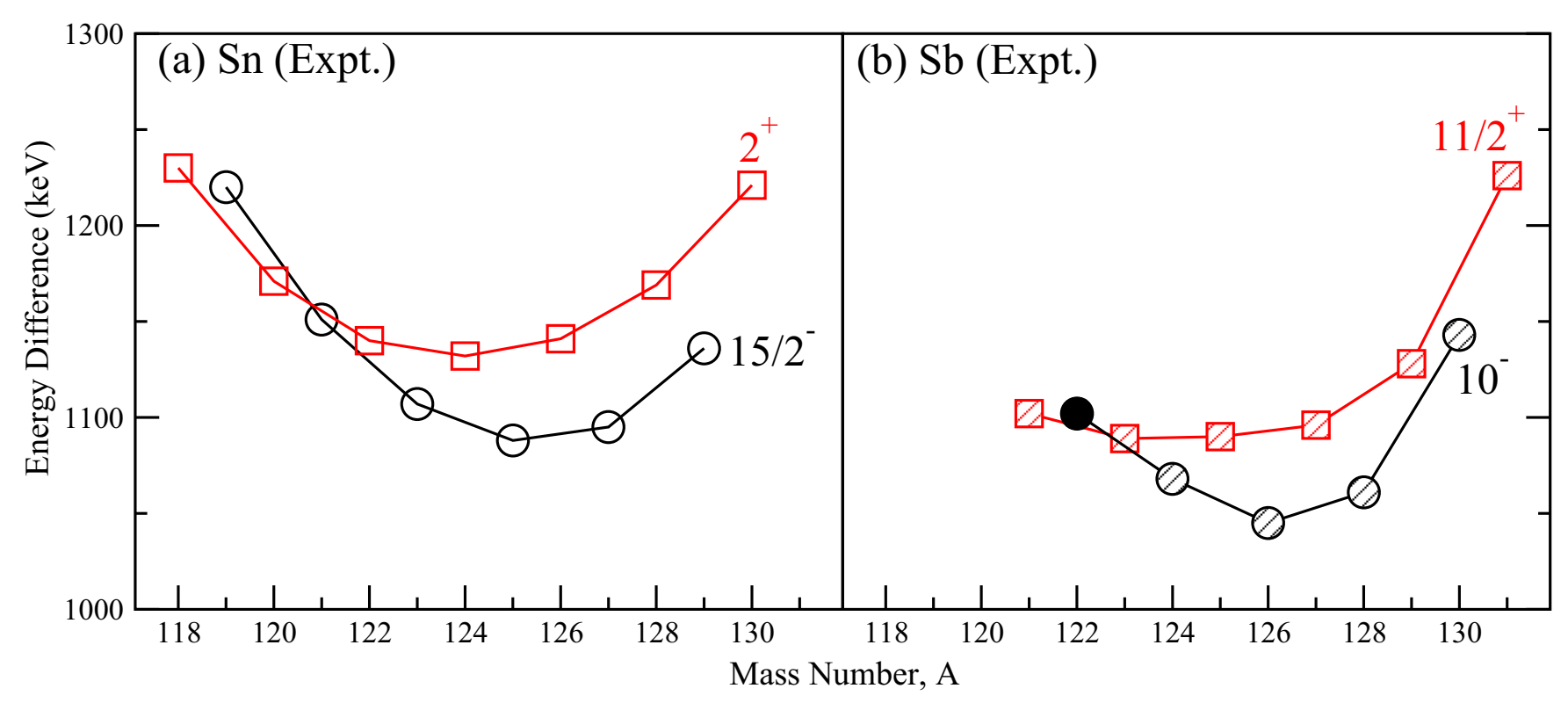

FIG. 12. Evolution of the experimental (Expt.) energy differences, as a function of mass number, in $\mathrm{Sn}($ open symbols $)(\mathrm{a}) E\left(2^{+}\right)-E\left(0^{+}\right)$ (red squares), $E\left(15 / 2^{-}\right)-E\left(11 / 2^{-}\right)$(black circle), and in Sb (shaded symbols) (b) $E\left(11 / 2^{+}\right)-E\left(7 / 2^{+}\right)\left(\right.$red squares), $E\left(10^{-}\right)-E\left(8^{-}\right)$ (black circle). The filled symbol is the newly observed state from the present experiment.

A better understanding of the aforementioned correspondence for the high-spin states in $\mathrm{Sb}$ and $\mathrm{Sn}$ isotopes was achieved by performing shell-model calculations, with the interaction used in Ref. [6] (denoted by SM1). The model space was constituted of a restricted single-particle space consisting of active particles, the neutrons in (v) $d_{3 / 2}, s_{1 / 2}, h_{11 / 2}$ orbits, and a proton in $(\pi) g_{7 / 2}$ orbit near the Fermi surface. This interaction was derived from the original $\mathrm{jj} 55 \mathrm{pn}$ interaction
[45], where the diagonal two-body matrix elements (TBMEs) were adjusted to account for the missing correlations in the restricted model space. The modified (original) multiplets, used in Ref. [6], were:

(i) $\left\langle d_{3 / 2}^{2} ; I|\hat{\mathcal{H}}| d_{3 / 2}^{2} ; I\right\rangle=-0.6048(-0.3024)$, and $0.22104(0.14814)$ for $I=0$ and 2 , respectively;

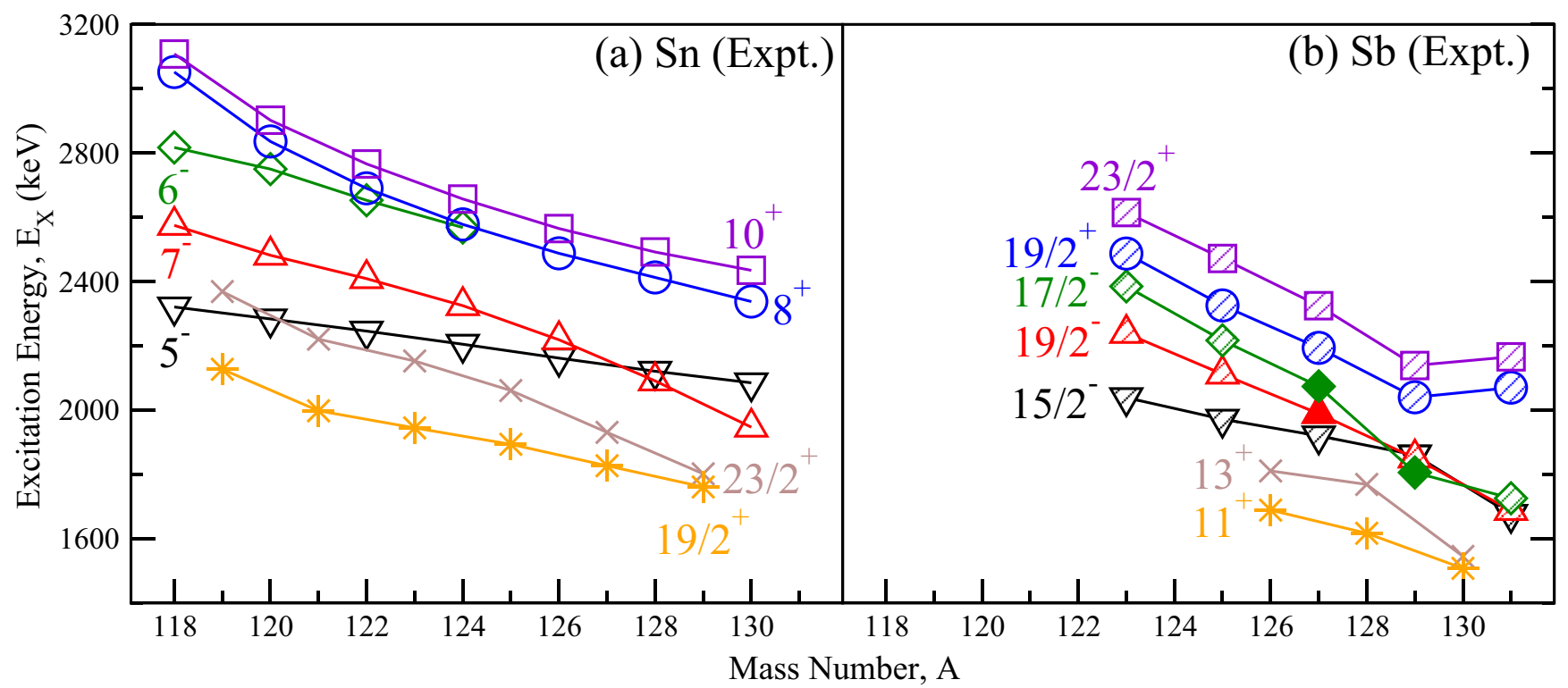

FIG. 13. Evolution of the experimental (Expt.) (a) $5^{-}$(black triangle down), $6^{-}$(green diamond), $7^{-}$(red triangle up), $8^{+}$(blue circle), $10^{+}$(violet square), $23 / 2^{+}$(brown x), and $19 / 2^{+}$(orange star) states in ${ }^{118-130} \mathrm{Sn}$ (open symbols), and (b) $15 / 2^{-}$(black triangle down), 17/2(green diamond), $19 / 2^{-}$(red triangle up), $19 / 2^{+}$(blue circle), $23 / 2^{+}$(violet square), $13^{+}$(brown x), and $11^{+}$(orange star) states in ${ }^{123-131} \mathrm{Sb}$ (shaded symbols) with mass number, A. The filled symbols are the newly observed states from the present experiment. 

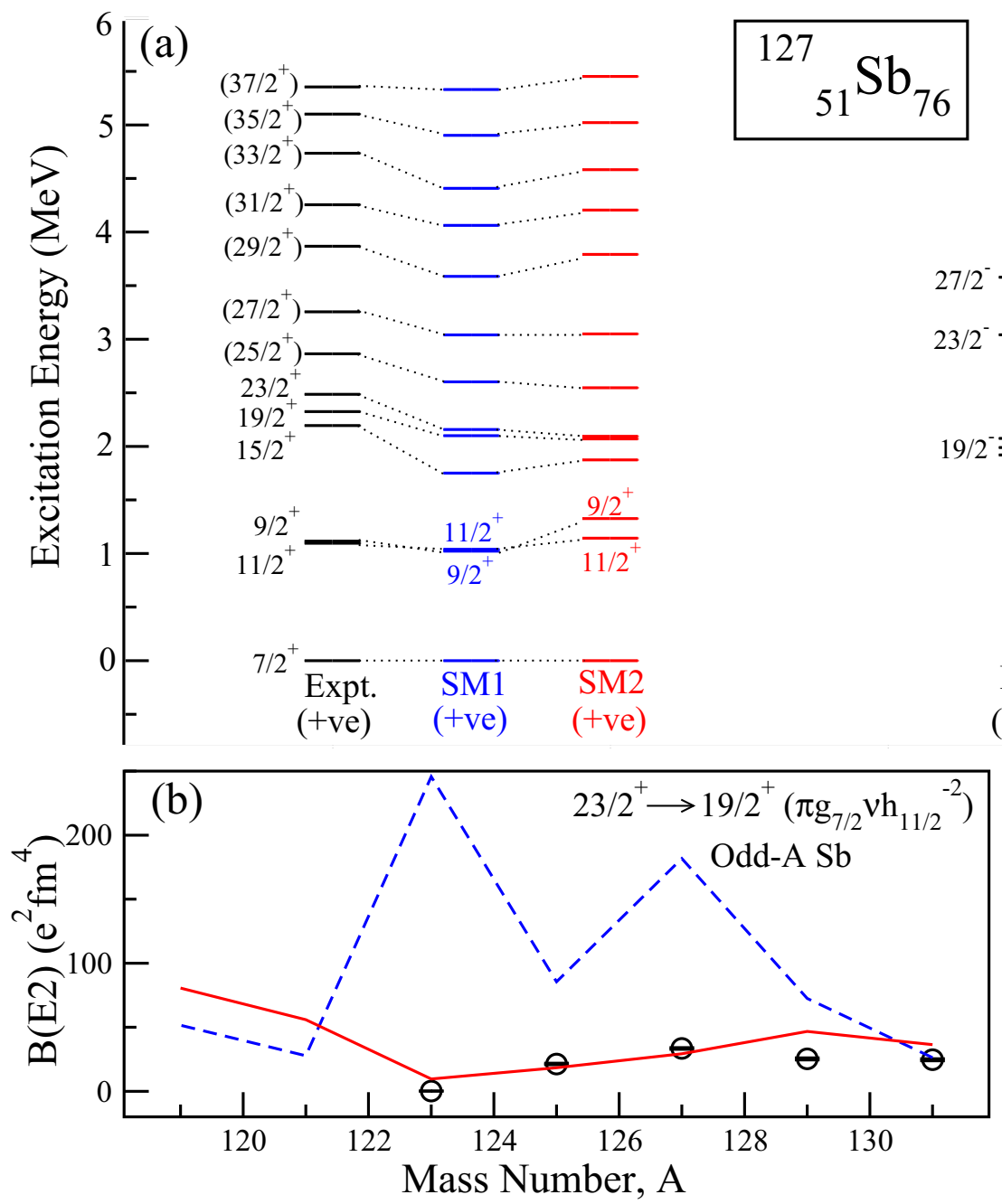

FIG. 14. (a) Comparison of the experimental (Expt.) level schemes with that obtained from shell model calculations using the interaction in Ref. [6] (SM1 blue) and the modified interaction in the present work (SM2 red) for both positive and negative-parity states in ${ }^{127} \mathrm{Sb}$. The different states with the same spin have been joined by dotted lines to show the agreement. (b) The experimental and calculated $\mathrm{B}(E 2)$ values (black square) for the $23 / 2^{+} \rightarrow 19 / 2^{+}$in odd- $A^{123-131} \mathrm{Sb}$ (see text).

(ii) $\left\langle s_{1 / 2}^{2} ; I|\hat{\mathcal{H}}| s_{1 / 2}^{2} ; I\right\rangle=-0.959904(-0.59994) \quad$ for $I=0$

(iii) $\left\langle h_{11 / 2}^{2} ; I|\hat{\mathcal{H}}| h_{11 / 2}^{2} ; I\right\rangle=-1.88544(-0.94545)$,

-0.88533 (-0.435330), 0.20683 (0.10683), 0.27145 (0.17145), and $0.33148(0.23148)$ for $I=0,2,6,8$, and 10 , respectively;

(iv) $\left\langle d_{3 / 2} h_{11 / 2} ; I|\hat{\mathcal{H}}| d_{3 / 2} h_{11 / 2} ; I\right\rangle=-0.08335(0.016650)$ for $I=4 ;$ and

(v) $\left\langle d_{3 / 2} s_{1 / 2} ; I|\hat{\mathcal{H}}| d_{3 / 2} s_{1 / 2} ; I\right\rangle=0.05607$ (-0.06399), for $I=2$

where $\hat{\mathcal{H}}$ represents the Hamiltonian. Effective charges of $e_{\nu}=0.9$ and $e_{\pi}=1.8$ were chosen to reproduce the known $B(E 2)$ values in ${ }^{130} \mathrm{Sn}\left(B(E 2) ; 10^{+} \rightarrow 8^{+}\right)$and ${ }^{134} \mathrm{Te}\left(B(E 2) ; 6^{+} \rightarrow 4^{+}\right)$isotopes. The calculations were performed by using the shell-model code, NATHAN [46]. Using SM1, the excitation energies could be well reproduced, while the binding energies and $B(E 2)$ transition probabilities were not properly reproduced. This is illustrated for ${ }^{127} \mathrm{Sb}$ for the level energies and for the $B\left(E 2 ; 23 / 2^{+} \rightarrow 19 / 2^{+}\right)$in odd- $A$ $\mathrm{Sb}$ isotopes in Fig. 14. Similar inconsistencies were observed for the other isomeric transitions (not shown in the figure).

Therefore, to improve the agreement of $B(E 2)$ values, the following parameters of the shell-model interaction were modified (denoted by SM2):

(i) the monopole part of $\left\langle h_{11 / 2}^{2}|\hat{\mathcal{H}}| h_{11 / 2}^{2}\right\rangle$ was reduced by $185 \mathrm{keV}$, which led to an improved reproduction of the binding energies and the $\left(B(E 2) ; 10^{+} \rightarrow 8^{+}\right)$for the even- $A \mathrm{Sn}$ isotopes;

(ii) the monopole part of $\left\langle d_{3 / 2}^{2}|\hat{\mathcal{H}}| d_{3 / 2}^{2}\right\rangle$ was reduced by $450 \mathrm{keV}$, which led to a better reproduction of the $\left(B(E 2) ; 23 / 2^{+} \rightarrow 19 / 2^{+}\right)$in odd- $A$ Sb isotopes;

(iii) the pairing term, $\left\langle d_{3 / 2}^{2}|\hat{\mathcal{H}}| h_{11 / 2}^{2}\right\rangle$, was increased by $260 \mathrm{keV}$, which led to the agreement of all the $B(E 2)$ values, except those for the odd- $A$ Sb isotopes. Also, all the excitation energies became higher by $2 \mathrm{MeV}$; 


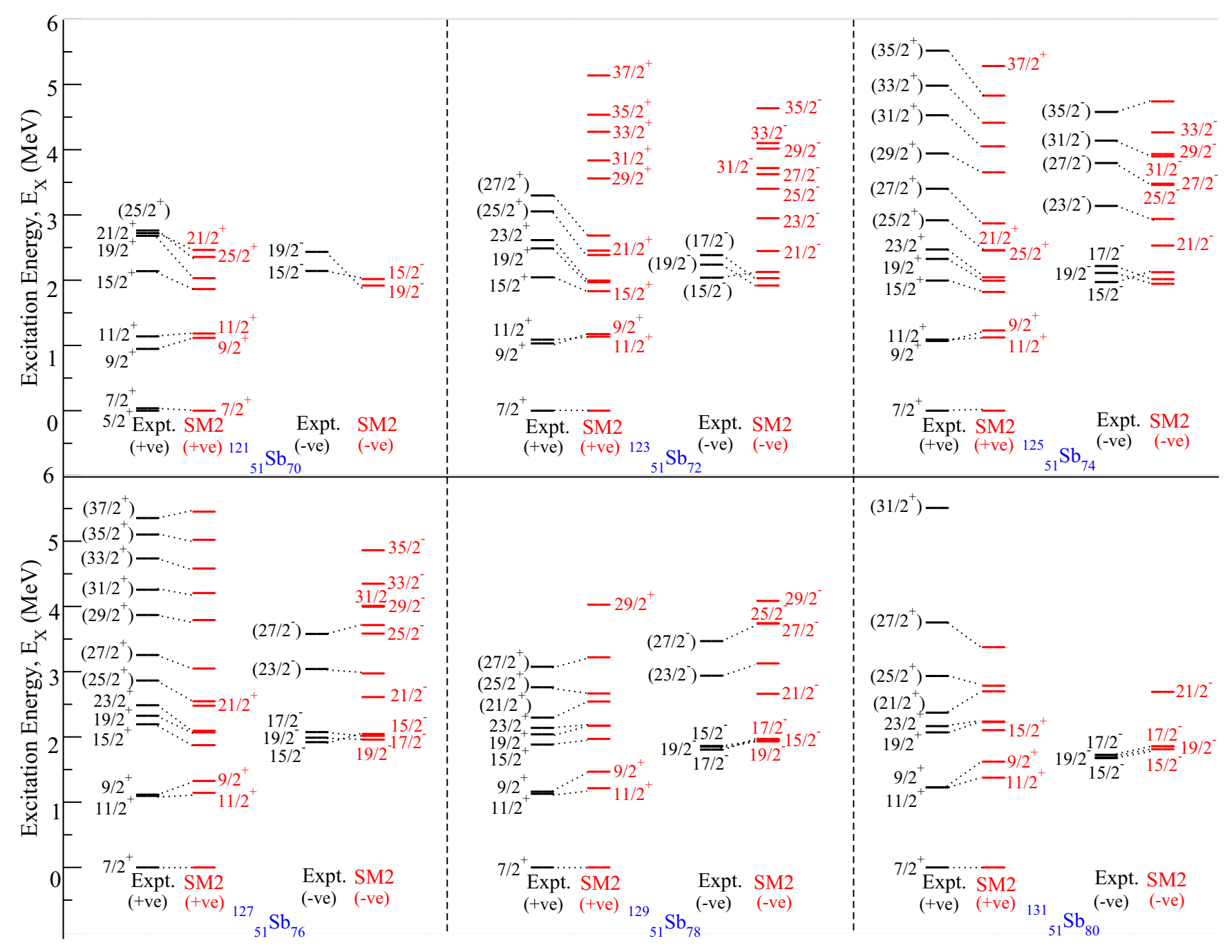

FIG. 15. Comparison of experimental (Expt.) (shown in black) [22-26] and theoretical calculations [from shell model (SM2); shown in red] level schemes for odd $-A{ }^{121-131} \mathrm{Sb}$ isotopes for both positive (+ve) and negative (-ve) parities. The experimental and shell-model states of the same spin-parity have been joined with dotted lines to show the agreement.

(iv) the pairing term, $\left\langle h_{11 / 2}^{2}|\hat{\mathcal{H}}| h_{11 / 2}^{2}\right\rangle$, was increased by $140 \mathrm{keV}$, to compensate for this increase in excitation energies as well as seeing to it that the $B(E 2)$ s of all the $\mathrm{Sn}$ and $\mathrm{Sb}$ do not change drastically;

(v) the $\left\langle\pi g_{7 / 2} v h_{11 / 2} ; I|\hat{\mathcal{H}}| \pi g_{7 / 2} v h_{11 / 2} ; I\right\rangle(I=8)$ was reduced by $400 \mathrm{keV}$, which reproduced the $B(E 2)$ s for the odd- $A$ Sb as well; and

(vi) the monopole part of $\left\langle d_{3 / 2} h_{11 / 2}|\hat{\mathcal{H}}| d_{3 / 2} h_{11 / 2}\right\rangle$ was increased by $30 \mathrm{keV}$ to achieve a better reproduction of $B(E 2)$ in odd- $A \mathrm{Sn}$ isotopes.

These modifications led to the reproduction of the binding energies (within $\sim 2 \mathrm{MeV}$ ), excitation energies (within $\sim 500 \mathrm{keV}$ ) and the $B(E 2)$ transition strengths (within $\sim 50 e^{2} \mathrm{fm}^{4}$ ) for the isotopic chains of ${ }^{119-130} \mathrm{Sn}$ and ${ }^{121-131} \mathrm{Sb}$. The energies of the excited states for ${ }^{127} \mathrm{Sb}$ and the improved $B(E 2)$ values for the odd $A \mathrm{Sb}$ isotopes are shown in Fig. 14. The reason behind the large discrepancy in the $B(E 2)$ s between SM1 and SM2 can be understood based on the difference in the wave functions obtained in both calculations.
The wave functions from SM2 are much more fragmented configuration-wise than those in SM1, leading to the dramatic reduction in the $B(E 2)$ values.

\section{A. Excitation energies}

A comparison of the experimental (black) and calculated (red) level schemes (using SM2) for both the positive (+ve) and negative (-ve) parities in odd- $A{ }^{123-131} \mathrm{Sb}$ isotopes is shown in Fig. 15. The data for ${ }^{121} \mathrm{Sb}$ are taken from Ref. [23] and are also shown in this figure for comparison. The calculated order for the $9 / 2^{+}$and $11 / 2^{+}$states are inverted only for the odd $-A^{123-125} \mathrm{Sb}$ isotopes. A mismatch in the ordering of the $15 / 2^{-}, 17 / 2^{-}$, and $19 / 2^{-}$states are observed in ${ }^{121-129} \mathrm{Sb}$ isotopes, as these states are very close in energy. Similarly, Fig. 16 shows the comparison of the experimental (black) and calculated (red) level schemes for both the positive ( + ve) and negative (-ve) parities in even- $A{ }^{122-130} \mathrm{Sb}$ isotopes. The data for ${ }^{120} \mathrm{Sb}$ are taken from Ref. [47] and are also shown in this figure for comparison. This figure shows that the experimental 


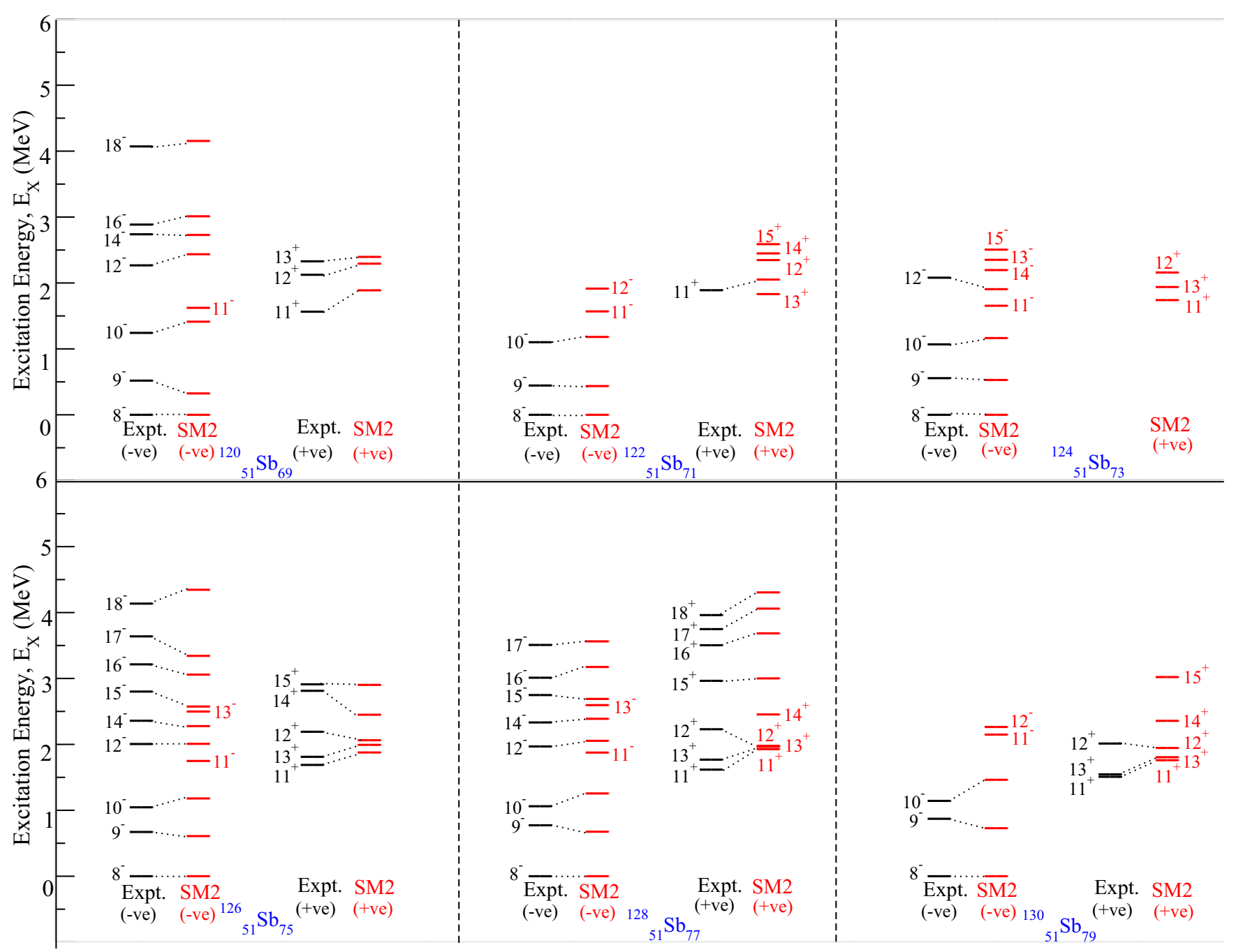

FIG. 16. Comparison of experimental (Expt.) (shown in black) $[6,35,36,43,47]$ and theoretical calculations [from shell model (SM2); shown in red] level schemes for even- $A^{122-130} \mathrm{Sb}$ isotopes for both positive (+ve) and negative (-ve) parities. The experimental and shell-model states of the same spin-parity are joined with dotted lines to show the agreement.

energies are in reasonable agreement with the theoretical calculations.

Isomeric $15 / 2^{-}, 19 / 2^{-}$, and $23 / 2^{+}$were previously observed in odd $-A \mathrm{Sb}$ isotopes. These were interpreted as the odd $g_{7 / 2}$ proton coupled to the known isomeric $5^{-}, 7^{-}$, and $10^{+}$states in the corresponding even- $(A-1)$ Sn isotopes, respectively [40]. These states along with the $6^{-}$and $8^{+}$ states for even- $A$ Sn and the $17 / 2^{-}$and $19 / 2^{+}$states for odd- $A \mathrm{Sb}$ are shown in Figs. 13(a) and 13(b), respectively. In addition, the evolution of $19 / 2^{+}$and $23 / 2^{+}$states in odd$A \mathrm{Sn}$, and $11^{+}$and $13^{+}$states in even- $A \mathrm{Sb}$ are shown in these plots. The newly identified $\left(17 / 2^{-}\right)$and $\left(19 / 2^{-}\right)$states in ${ }^{127} \mathrm{Sb}$; and the $\left(17 / 2^{-}\right)$state in ${ }^{129} \mathrm{Sb}$ (from the current experimental analysis) fit in the systematics. Comparison with the $\mathrm{Sn}$ isotopes shows that the energies of the positive-parity states in $\mathrm{Sb}$ follow a pattern similar to that of the Sn isotopes, with a slight dip in the case of ${ }^{129} \mathrm{Sb}$. Also, the crossing of the $7^{-}$and $5^{-}$observed at ${ }^{128} \mathrm{Sn}$ is seen in the equivalent states in ${ }^{129} \mathrm{Sb}$. In addition, the newly identified $11^{+}$and $13^{+}$ states in even- $A \mathrm{Sb}$ isotopes follow a similar pattern to the corresponding $19 / 2^{+}$and $23 / 2^{+}$states in odd- $A$ Sn isotopes.

\section{B. $B(E 2)$ transition probabilities}

The experimental $B(E 2)$ values along with SM2 shellmodel calculations are shown in Fig. 17.

Case I: (a) $10^{+} \rightarrow 8^{+}$in even- $A^{118-130} \mathrm{Sn}[16,21,48]$, (b) $23 / 2^{+} \rightarrow 19 / 2^{+}$in odd $-A^{123-131} \mathrm{Sb}$ (see text);

Case II: (c) $7^{-} \rightarrow 5^{-}$in even- $A{ }^{118-126} \mathrm{Sn} \mathrm{[21],} \mathrm{(d)}$ $19 / 2^{-} \rightarrow 15 / 2^{-}$in odd- $A^{123-131} \mathrm{Sb}$ (see text);

Case III: (e) $23 / 2^{+} \rightarrow 19 / 2^{+}$in odd- $A^{119-129} \mathrm{Sn}[20,21]$, (f) $13^{+} \rightarrow 11^{+}$states in the even- $A{ }^{126-130} \mathrm{Sb}$ (see text); and

Case IV: $\left(\right.$ g) $27 / 2^{-} \rightarrow 23 / 2^{-}$in odd- $A^{119-129} \mathrm{Sn}$ isotopes $[20,21,49]$.

The full calculations are shown by red solid lines, those restricted to one broken pair are shown by dash-dotted 


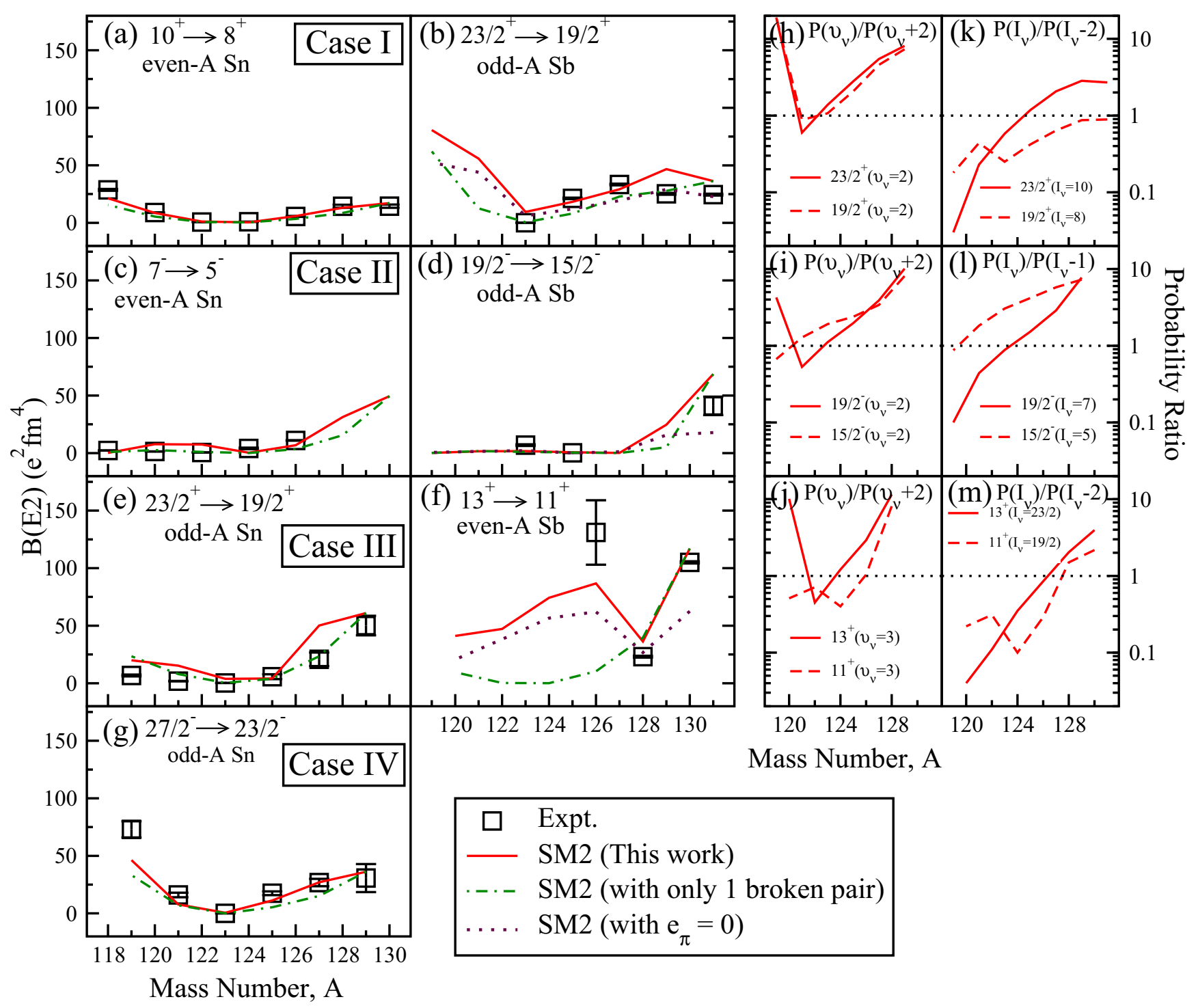

FIG. 17. The experimental (Expt.) $\mathrm{B}(E 2)$ values (black squares) for the (a) $10^{+} \rightarrow 8^{+}$in even- $A^{118-130} \mathrm{Sn}[16,21,48],(\mathrm{b}) 23 / 2^{+} \rightarrow 19 / 2^{+}$ in odd- $A{ }^{123-131} \mathrm{Sb}$ (see text), (c) $7^{-} \rightarrow 5^{-}$in even- $A^{118-126} \mathrm{Sn}[21]$, (d) $19 / 2^{-} \rightarrow 15 / 2^{-}$transitions in odd- $A{ }^{123-131} \mathrm{Sb}$ (see text), (e) $23 / 2^{+} \rightarrow 19 / 2^{+}$in odd- $A^{119-129} \mathrm{Sn}[20,21]$, (f) $13^{+} \rightarrow 11^{+}$in the even- $A^{126-130} \mathrm{Sb}$ (see text), and (g) $27 / 2^{-} \rightarrow 23 / 2^{-}$in odd- $A^{119-129} \mathrm{Sn}$ isotopes $[20,21,49]$. The shell-model calculations using the full interaction SM2 (red solid line), SM2 restricted to only one broken neutron pair (green dash-dotted line) and SM2 with $e_{\pi}=0$ (purple dotted line) are also shown. The probability ratios for the (h)-(j) neutron seniority mixing $\left[\left(P\left(v_{v}\right) / P\left(v_{v}+2\right)\right]\right.$ and $(\mathrm{k})-(\mathrm{m})$ neutron angular-momentum mixing $\left[\left(P\left(I_{v}\right) / P\left(I_{v}-2\right)\right]\right.$ and $\left[\left(P\left(I_{v}\right) / P\left(I_{v}-1\right)\right]\right.$ are also shown.

green lines, and the full calculations with proton effective charge, $e_{\pi}=0$, are shown by dotted violet lines. The seniority mixing, represented by the ratio of probabilities of lowest, natural $\left(v_{v}\right)$, and higher $\left(v_{v}+2\right)$ neutron seniorities $\left[P\left(v_{v}\right) / P\left(v_{v}+2\right)\right]$, are shown in Figs. 17(h)-17(j) for Cases I-III, respectively. The dotted black line with $P\left(v_{v}\right) / P\left(v_{v}+2\right)=1$ represents that the mixing of seniorities, $v_{v}$ and $v_{v}+2$, are equal. Similarly, Figs. $17(\mathrm{k})-17(\mathrm{~m})$ for Cases I-III, respectively, denote the neutron angularmomentum mixing, represented by the ratio of probabilities of highest $\left(I_{v}\right)$ and lower $\left(I_{v}-2\right.$ or $\left.I_{v}-1\right)$ neutron angular momenta $\left[P\left(I_{v}\right) / P\left(I_{v}-2\right)\right.$ or $\left.P\left(I_{v}\right) / P\left(I_{v}-1\right)\right]$. The dotted black line, similar to the case of neutron seniorities, denotes that the mixing of $I_{v}$ and $I_{v}-2$ or $I_{v}-1$ are equal.
The present calculations agree well with the experimental values and the experimental trends are reproduced. In general, the shape of the $B(E 2)$ curves for Sn follow a parabolic behavior, as expected for seniority scheme. The full calculation and the one restricted to one broken pair, in Sn, are very similar and also the calculated neutron seniority $v_{v}=2,3$ dominates for odd $A$, even- $A \mathrm{Sn}$, respectively. In addition, the behavior of $B(E 2)$ for odd- $A \mathrm{Sb}$ isotopes is similar to that of the corresponding $\mathrm{Sn}$, except the even- $A \mathrm{Sb}$. The proton charge $\left(e_{\pi}\right)$ does not have a considerable impact on the $B(E 2)$, except in even- $A \mathrm{Sb}$. A detailed discussion of the four different cases, mentioned above, is given below:

Case I: From shell-model calculations, it is seen that the dominant configuration of the $10^{+}$and $8^{+}$states in 

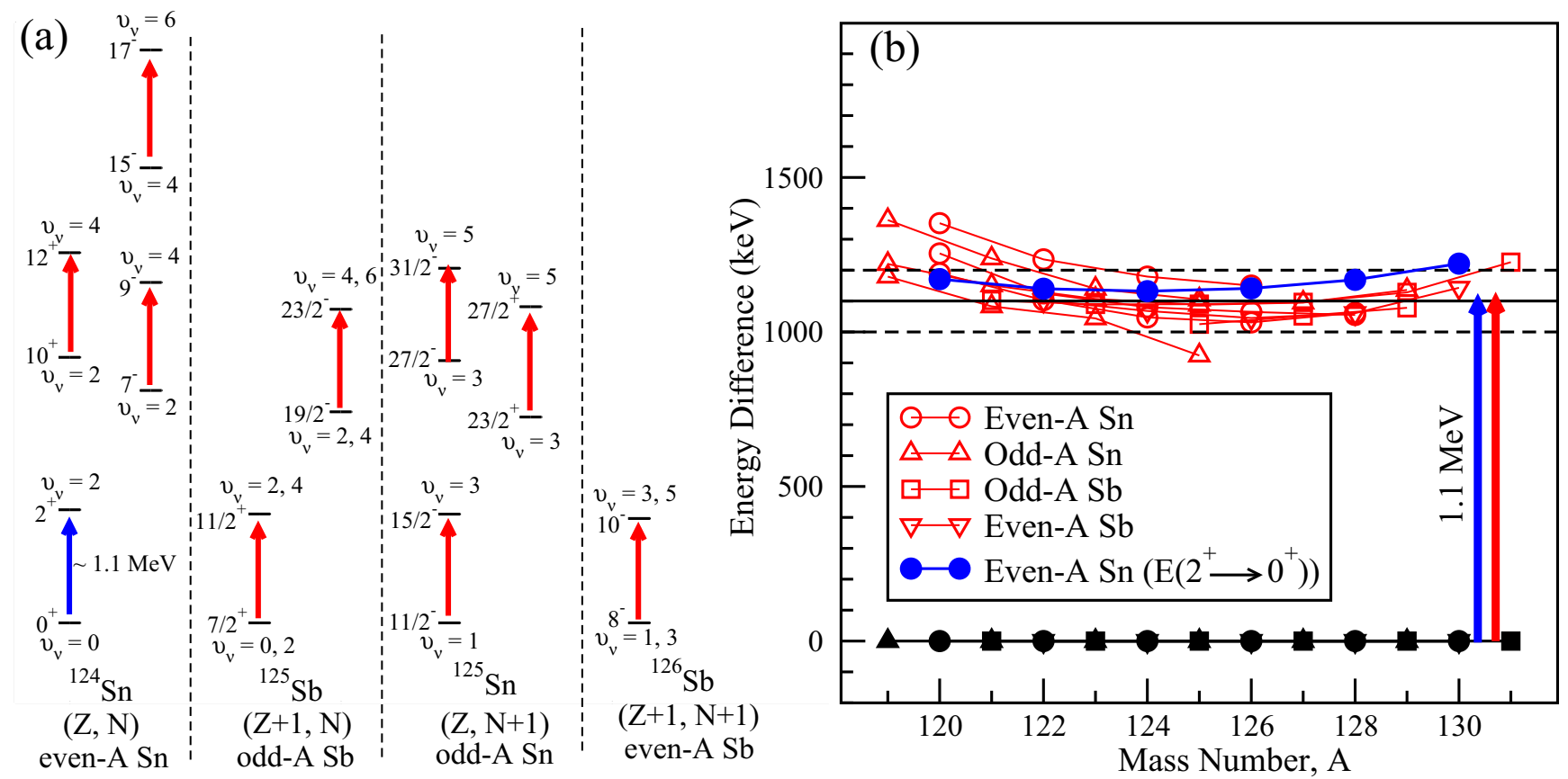

FIG. 18. (a) The states with different neutron seniorities at different excitation energies in even- $A \mathrm{Sn}(Z, N)\left({ }^{124} \mathrm{Sn}\right)$, odd- $A$ Sb $(Z+1, N)$ $\left({ }^{125} \mathrm{Sb}\right)$, odd- $A \mathrm{Sn}(Z, N+1)\left({ }^{125} \mathrm{Sn}\right)$, and even- $A \mathrm{Sb}(Z+1, N+1)\left({ }^{126} \mathrm{Sb}\right)$ isotopes. The blue and red arrows represent the energy required to break the first pair of neutrons from seniority $v_{v}=n$ to $v_{v}=n+2(n=0, \ldots, 4)$ in even- $A$ Sn $\left[E\left(2^{+} \rightarrow 0^{+}\right)\right]$and other isotopes, respectively. (b) Plot of the energy differences between states with neutron seniority $v_{v}=n$ and $v_{v}=n+2(n=0, \ldots, 4)$, as shown in panel (a), for odd- $A$ and even- $A{ }^{119-130} \mathrm{Sn}$ and ${ }^{121-131} \mathrm{Sb}$ nuclei.

even- $A$ Sn is $v h_{11 / 2}^{-2}$, while that of the $23 / 2^{+}$and $19 / 2^{+}$ states in odd- $A \mathrm{Sb}$ is $\pi g_{7 / 2} v h_{11 / 2}^{-2}$. The nature of the curves in Figs. 17(a) and 17(b) is not completely identical. This is evident from the differences in the full calculations and that restricted to one broken pair in Sb. For a microscopic understanding behind this difference, a comparison of the neutron seniority for the $23 / 2^{+}$and $19 / 2^{+}$states was carried out. Figure 17(h) shows that the lowest neutron seniority $v_{v}=2$ is mixed with $v_{v}=4$ for both states. The mixing increases with the increasing number of valence neutron holes. However, for the lowest $A$, the number of available neutron pairs is reduced in the restricted model space, which leads to a sudden drastic decrease in seniority mixing. In addition to mixing of neutron seniorities, neutron angularmomentum mixing is also observed, as shown in Fig. 17(k). The angular-momentum mixing also increases with increase in the number of valence neutron holes. A comparison of neutron seniority and neutron angular-momentum mixing plots shows that the angular-momentum mixing is the dominant one.

Case II: Shell-model calculations show that the dominant configuration of the $7^{-}$and $5^{-}$states in even- $A \mathrm{Sn}$ is $v h_{11 / 2}^{-1} d_{3 / 2}^{-1}$, while that of the $19 / 2^{-}$and $15 / 2^{-}$states in odd- $A$ $\mathrm{Sb}$ is $\pi g_{7 / 2} \nu h_{11 / 2}^{-1} d_{3 / 2}^{-1}$. The nature of the curves in Figs. 17(c) and 17 (d) is similar. A comparison of the neutron seniority for the $19 / 2^{-}$and $15 / 2^{-}$states was carried out. Figure 17(i) shows that the lowest neutron seniority $v_{\nu}=2$ is mixed with the $v_{v}=4$ for both states, similar to Case I. In addition to mixing of neutron seniorities, neutron angular-momentum mixing is also observed, as shown in Fig. 17(1), which is of similar order as the seniority mixing. The angular-momentum mixing also increases with increasing number of valence neutron holes.

Case III: The shell-model calculations show that the dominant configuration of the $23 / 2^{+}$and $19 / 2^{+}$states in odd- $A$ Sn is $v h_{11 / 2}^{-2} d_{3 / 2}^{-1}$, while that of the $13^{+}$and $11^{+}$states in even- $A$ $\mathrm{Sb}$ is $\pi g_{7 / 2} \nu h_{11 / 2}^{-2} d_{3 / 2}^{-1}$. The nature of the curves in Figs. 17(e) and $17(\mathrm{f})$ is significantly different. This is also evident from the differences in the full calculations and that restricted to one broken pair in Sb. The calculations with proton charge restricted to zero show that the neutrons have a considerable impact on the $B(E 2)$ in even- $A$ Sb. For a microscopic understanding behind this difference, a comparison of the neutron seniority for the $23 / 2^{+}$and $19 / 2^{+}$states was carried out. Figure 17(j) shows that the lowest neutron seniority $v_{v}=3$ is mixed with the $v_{v}=5$ for both states. The mixing increases with increasing number of valence neutron holes. In addition to mixing of neutron seniorities, neutron angular-momentum mixing is also observed, as shown in Fig. 17(m). The angularmomentum mixing also increases with increasing number of valence neutron holes. A comparison of neutron seniority and neutron angular-momentum mixing plots show that the angular-momentum mixing is the dominant one, just as in Case I. Note that, around $A=126$, as compared with the earlier two cases, both the seniority and angular-momentum mixing are significant; the higher seniority $\left(v_{v}+2\right)$ and the lower angular momentum $\left(I_{\nu}-2\right)$ dominate the $11^{+}$and $13^{+}$ states. 
Case IV: The shell-model calculations show that the dominant configuration of the $27 / 2^{-}$and $23 / 2^{-}$states in odd- $A$ Sn is $v h_{11 / 2}^{-3}$. The corresponding isomer in even- $A \mathrm{Sb}$ with configuration $\pi g_{7 / 2} v h_{11 / 2}^{-3}$ is expected at $I^{\pi}=16^{-}$. In the present work, the $16^{-}$states in ${ }^{126,128} \mathrm{Sb}$ decay by $M 1$ transitions to $15^{-}$states, and hence no isomer was observed. In ${ }^{120} \mathrm{Sb}$, in contrast, the $16^{-}$state decays via a $148 \mathrm{keV}$ transition to the $14^{-}$state and has a half-life $T_{1 / 2}=14(3) \mathrm{ns}$ [47]. The nature of the curve in Fig. 17(g) is a parabola, similar to Fig. 17(a). The present calculations show that the dominant seniority for these states is $v_{v}=3$, which in fact is in agreement with the calculations assuming only one broken pair.

\section{Neutron pair-breaking energies}

A striking feature of the constancy of the energy differences, where the increase in the number of broken neutron pairs is involved, was observed and is shown in Fig. 18. Figure 18(a) shows particular states in even- $A$ Sn $(Z, N)\left({ }^{124} \mathrm{Sn}\right)$, and odd- $A \mathrm{Sn}(Z, N+1)\left({ }^{125} \mathrm{Sn}\right)$ isotopes, which involve the breaking of a pair of neutrons, leading to increase in seniority from $v_{v}=n$ to $v_{v}=n+2$, where $n=0, \ldots, 4$ at different excitation energies. Similar states in odd- $A \mathrm{Sb}(Z+1, N)$ $\left({ }^{125} \mathrm{Sb}\right)$ and even- $A \mathrm{Sb}(Z+1, N+1)\left({ }^{126} \mathrm{Sb}\right)$ isotopes are also shown in the same figure. All the arrows represent the energy difference quantum with magnitude of $\sim 1.1 \mathrm{MeV}$. The blue arrow represents the breaking of the first pair of neutrons in even- $A \mathrm{Sn}$ for the $E\left(2^{+} \rightarrow 0^{+}\right)$. The red arrows are for all the other transitions. A plot of the energy differences in ${ }^{119-130} \mathrm{Sn}$ and ${ }^{121-131} \mathrm{Sb}$ isotopes is shown in Fig. 18(b). A subset of this figure, including only the lower spins, was shown earlier in Fig. 12. This figure shows that the average energy for the breaking of the first and second pair of neutrons is $\sim 1.1 \mathrm{MeV}$, and this is constant (with a deviation of $\sim 100 \mathrm{keV}$ ) for a wide range of mass numbers, irrespective of the excitation energy and mixing of neutron seniorities $\left(v_{v}\right)$ in the case of Sn and $\mathrm{Sb}$. In addition, it follows the behaviour of even- $A$ Sn isotopes for $E\left(2^{+} \rightarrow 0^{+}\right)$.

\section{SUMMARY AND CONCLUSIONS}

The neutron-rich ${ }^{122-131} \mathrm{Sb}$ isotopes were produced as fission fragments in the reaction ${ }^{9} \mathrm{Be}\left({ }^{238} \mathrm{U}, f\right)$ with 6.2 $\mathrm{MeV} / \mathrm{u}$ beam energy. The isomers already known in the odd- $A{ }^{123-131} \mathrm{Sb}$ isotopes were confirmed and a number of new prompt and delayed transitions were identified for all these isotopes. New isomers and prompt and delayed $\gamma$ rays were identified in the even- $A{ }^{122-130} \mathrm{Sb}$ isotopes. These results could be achieved by using the unique combination of the AGATA, VAMOS++, and EXOGAM detectors, which was used for the prompt-delayed spectroscopy of fission fragments. A good agreement was achieved between the experiment and the results of the shell-model calculations in the restricted model space using an optimized interaction. Also, the level schemes were in good agreement with that of the corresponding states in the $\mathrm{Sn}$ isotopes. The presence of a single valence proton particle in the $g_{7 / 2}$ orbital leads to a neutron seniority mixing and neutron angular-momentum mixing in the $\mathrm{Sb}$ isotopes for all the states. The angular-momentum mixing, in general, was found to be significantly stronger than the seniority mixing. This work shows that further experimental work is required to search for new isomers in the even- $A{ }^{122,124} \mathrm{Sb}$ isotopes, which could not be observed in this work either due to low statistics, too long half-life, or too short half-life. This will give more insight into the nature of the $B(E 2)$ values, and hence the mixing of seniorities and angular momenta due to the $\nu \pi$ interaction.

\section{ACKNOWLEDGMENTS}

The authors would like to thank the AGATA Collaboration for the availability of the AGATA $\gamma$-ray tracking array at GANIL. We acknowledge the important technical contributions of GANIL accelerator staff. We thank C. Schmitt and A. O. Macchiavelli for help in various aspects of data collection. We also thank P. Van Isacker for his valuable discussions on the theoretical interpretation and careful reading of the paper. P.B. and A.M. acknowledge support from the Polish National Science Centre (NCN) under Contract No. 2016/22/M/ST2/00269 and the French LEA COPIGAL project. S.Bi., R.B., S.Bh., S.Bh., and R.P. acknowledge support from CEFIPRA project No. 5604-4 and the LIA FranceIndia agreement. H.L.C. and P.F. acknowledge support from the U.S. Department of Energy, Office of Science, Office of Nuclear Physics under Contract No. DE-AC02-05CH11231 (LBNL). R.M.P.V. acknowledge partial support by Ministry of Science, Spain, under the grants BES-2012-061407, SEV2014-0398, FPA2017-84756-C4 and by EU FEDER funds. A.J. was supported by the Spanish Ministerio de Economía y Competitividad under contract FPA2017-84756-C4-2-P.
[1] R. F. Casten, Nuclear Structure from a Simple Perspective (Oxford Science Publications, Oxford, 2000).

[2] NUDAT2, http://www.nndc.bnl.gov/nudat2/ (2019).

[3] T. Togashi, Y. Tsunoda, T. Otsuka, N. Shimizu, and M. Honma, Phys. Rev. Lett. 121, 062501 (2018).

[4] B. Maheshwari and A. K. Jain, Phys. Lett. B 753, 122 (2016).

[5] A. I. Morales, G. Benzoni, H. Watanabe, G. de Angelis, S. Nishimura, L. Coraggio, A. Gargano, N. Itaco, T. Otsuka, Y. Tsunoda, P. Van Isacker, F. Browne et al., Phys. Lett. B 781, 706 (2018)
[6] M. Rejmund, A. Navin, S. Biswas, A. Lemasson, M. Caamaño, E. Clément, O. Delaune, F. Farget, G. de France, B. Jacquot, and P. Van Isacker, Phys. Lett. B 753, 86 (2016).

[7] P. Bhattacharyya, P. J. Daly, C. T. Zhang, Z. W. Grabowski, S. K. Saha, R. Broda, B. Fornal, I. Ahmad, D. Seweryniak, I. Wiedenhöver, M. P. Carpenter, R. V. F. Janssens et al., Phys. Rev. Lett. 87, 062502 (2001).

[8] P. Cottle, Nature (London) 465, 430 (2010).

[9] K. L. Jones, A. S. Adekola, D. W. Bardayan, J. C. Blackmon, K. Y. Chae, K. A. Chipps, J. A. Cizewski, L. Erikson, C. Harlin, 
R. Hatarik, R. Kapler, R. L. Kozub et al., Nature (London) 465, 454 (2010).

[10] D. Rosiak, M. Seidlitz, P. Reiter, H. Naïdja, Y. Tsunoda, T. Togashi, F. Nowacki, T. Otsuka, G. Colò, K. Arnswald, T. Berry, A. Blazhev et al., Phys. Rev. Lett. 121, 252501 (2018).

[11] L. Coraggio, A. Covello, A. Gargano, N. Itaco, and T. T. S. Kuo, Phys. Rev. C 91, 041301(R) (2015).

[12] E. Teruya, N. Yoshinaga, K. Higashiyama, and A. Odahara, Phys. Rev. C 92, 034320 (2015).

[13] S. Biswas, R. Palit, A. Navin, M. Rejmund, A. Bisoi, M. S. Sarkar, S. Sarkar, S. Bhattacharyya, D. C. Biswas, M. Caamaño, M. P. Carpenter, D. Choudhury et al., Phys. Rev. C 93, 034324 (2016).

[14] H.-K. Wang, S. K. Ghorui, K. Kaneko, Y. Sun, and Z. H. Li, Phys. Rev. C 96, 054313 (2017).

[15] Y. H. Kim, M. Rejmund, P. Van Isacker, and A. Lemasson, Phys. Rev. C 97, 041302(R) (2018).

[16] B. Fogelberg, K. Heyde and J. Sau, Nucl. Phys. A 352, 157 (1981).

[17] S. Pietri, A. Jungclaus, M. Górska, H. Grawe, M. Pfützner, L. Cáceres, P. Detistov, S. Lalkovski, V. Modamio, Z. Podolyák, P. H. Regan, D. Rudolph et al., Phys. Rev. C 83, 044328 (2011).

[18] A. Astier, M.-G. Porquet, C. Theisen, D. Verney, I. Deloncle, M. Houry, R. Lucas, F. Azaiez, G. Barreau, D. Curien, O. Dorvaux, G. Duchêne et al., Phys. Rev. C 85, 054316 (2012).

[19] W. Iskra, R. Broda, J. Wrzesinski, M. P. Carpenter, C. J. Chiara, B. Fornal, N. Hoteling, R. V. Janssens, F. G. Kondev, W. Królas, T. Lauritsen, T. Pawat et al., Act. Phys. Pol. B 89, 395 (2014).

[20] R. L. Lozeva, G. S. Simpson, H. Grawe, G. Neyens, L. A. Atanasova, D. L. Balabanski, D. Bazzacco, F. Becker, P. Bednarczyk, G. Benzoni, N. Blasi, A. Blazhev et al., Phys. Rev. C 77, 064313 (2008).

[21] L. W. Iskra, R. Broda, R. V. F. Janssens, C. J. Chiara, M. P. Carpenter, B. Fornal, N. Hoteling, F. G. Kondev, W. Królas, T. Lauritsen, T. Pawłat, D. Seweryniak et al., Phys. Rev. C 93, 014303 (2016).

[22] D. S. Judson, A. M. Bruce, T. Kibédi, G. D. Dracoulis, A. P. Byrne, G. J. Lane, K. H. Maier, C.-B. Moon, P. Nieminen, J. N. Orce, and M. J. Taylor, Phys. Rev. C 76, 054306 (2007).

[23] H. Watanabe, G. J. Lane, G. D. Dracoulis, T. Kibédi, A. P. Byrne, P. Nieminen, R. O. Hughes, F. G. Kondev, M. P. Carpenter, R. V. F. Janssens, T. Lauritsen, D. Seweryniak et al., Phys. Rev. C 79, 024306 (2009).

[24] H. Watanabe, G. J. Lane, G. D. Dracoulis, a. P. Byrne, P. Nieminen, F. G. Kondev, K. Ogawa, M. P. Carpenter, R. V. F. Janssens, T. Lauritsen, D. Seweryniak, S. Zhu et al., Eur. Phys. J. A 42, 163 (2009).

[25] J. Genevey, J. A. Pinston, H. R. Faust, R. Orlandi, A. Scherillo, G. S. Simpson, I. S. Tsekhanovich, A. Covello, A. Gargano, and W. Urban, Phys. Rev. C 67, 054312 (2003).

[26] J. Genevey, J. A. Pinston, H. Faust, C. Foin, S. Oberstedt, and M. Rejmund, Eur. Phys. J. A 9, 191 (2000).

[27] M. Rejmund, B. Lecornu, A. Navin, C. Schmitt, S. Damoy, O. Delaune, J. M. Enguerrand, G. Fremont, P. Gangnant, L. Gaudefroy, B. Jacquot, J. Pancin et al., Nucl. Instrum. Methods Phys. Res., Sect. A 646, 184 (2011).

[28] A. Navin, M. Rejmund, C. Schmitt, S. Bhattacharyya, G. Lhersonneau, P. van Isacker, M. Caamaño, E. Clément, O. Delaune, F. Farget, G. de France, and B. Jacquot, Phys. Lett. B 728, 136 (2014).
[29] A. Navin and M. Rejmund, Gamma-ray spectroscopy of neutron-rich fission fragments, in Yearbook of Encyclopedia of Science and Technology (McGraw-Hill, New York, 2014).

[30] S. Akkoyun, A. Algora, B. Alikhani, F. Ameil, G. de Angelis, L. Arnold, A. Astier, A. Ataç, Y. Aubert, C. Aufranc, A. Austin, S. Aydin et al., Nucl. Instrum. Methods Phys. Res., Sect. A 668, 26 (2012).

[31] M. Vandebrouck, A. Lemasson, M. Rejmund, G. Fremont, J. Pancin, A. Navin, C. Michelagnoli, J. Goupil, C. Spitaels, and B. Jacquot, Nucl. Instrum. Methods Phys. Res., Sect. A 812, 112 (2016).

[32] Y. H. Kim, A. Lemasson, M. Rejmund, A. Navin, S. Biswas, C. Michelagnoli, I. Stefan, R. Banik, P. Bednarczyk, S. Bhattacharya, S. Bhattacharyya, E. Clément et al., Eur. Phys. J. A 53, 162 (2017).

[33] J. Simpson, F. Azaiez, G. DeFrance, J. Fouan, J. Gerl, R. Julin, W. Korten, P. Nolan, B. Nyakó, G. Sletten, and P. Walker, Act. Phys. Hung. Ser. Heavy Ion Phys. 11, 159 (2000).

[34] T. Kibédi, T. Burrows, M. Trzhaskovskaya, P. Davidson, and C. Nestor, Nucl. Instrum. Methods Phys. Res., Sect. A 589, 202 (2008).

[35] W. F. van Gunsteren and D. Rabenstein, Z. Phys. A: At. Nucl. (1975) 282, 55 (1977).

[36] H. Langevin-Joliot, V. Datar, E. Gerlic, J. Van de Wiele, F. Azaiez, S. Fortier, S. Gales, J. Guillot, J. M. Maison, C. P. Massolo, G. Duhamel, and G. Perrin, Phys. Rev. C 38, 1168 (1988).

[37] M. G. Porquet, T. Venkova, R. Lucas, A. Astier, A. Bauchet, I. Deloncle, A. Prévost, F. Azaiez, G. Barreau, A. Bogachev, N. Buforn, A. Buta et al., Eur. Phys. J. A 24, 39 (2005).

[38] G. A. Jones, S. J. Williams, P. M. Walker, Z. Podolyák, S. Zhu, M. P. Carpenter, J. J. Carroll, R. S. Chakrawarthy, P. Chowdhury, I. J. Cullen, G. D. Dracoulis, A. B. Garnsworthy et al., Phys. Rev. C 77, 034311 (2008).

[39] J. J. Ressler, J. A. Caggiano, C. J. Francy, P. N. Peplowski, J. M. Allmond, C. W. Beausang, L. A. Bernstein, D. L. Bleuel, J. T. Burke, P. Fallon, A. A. Hecht, D. V. Jordan et al., Phys. Rev. C 81, 014301 (2010).

[40] K. E. Apt and W. B. Walters, Phys. Rev. C 9, 310 (1974).

[41] H. Huck, M. L. Pérez, and J. J. Rossi, Phys. Rev. C 26, 621 (1982).

[42] C. A. Stone and W. B. Walters, Z. Phys. A 328, 257 (1987).

[43] J. Genevey, J. A. Pinston, C. Foin, M. Rejmund, H. Faust, and B. Weiss, Phys. Rev. C 65, 034322 (2002).

[44] F. Schussler, J. Blachot, J. P. Bocquet, and E. Monnand, Z. Phys. A: At. Nucl. (1975) 281, 229 (1977).

[45] M. Hjorth-Jensen, T. T. Kuo, and E. Osnes, Phys. Rep. 261, 125 (1995).

[46] E. Caurier, G. Martinez-Pinedo, F. Nowacki, A. Poves, J. Retamosa, and A. P. Zuker, Phys. Rev. C 59, 2033 (1999).

[47] L. Liu, S. Y. Wang, Z. Q. Chen, C. Liu, B. Qi, D. P. Sun, S. Wang, Q. An, C. J. Xu, P. Zhang, Z. Q. Li, C. Y. Niu et al., Phys. Rev. C 90, 014313 (2014).

[48] R. Broda, R. H. Mayer, I. G. Bearden, P. Benet, P. J. Daly, Z. W. Grabowski, M. P. Carpenter, R. V. F. Janssens, T. L. Khoo, T. Lauritsen, E. F. Moore, S. Lunardi et al., Phys. Rev. Lett. 68, 1671 (1992).

[49] R. H. Mayer, D. T. Nisius, I. G. Bearden, P. Bhattacharyya, L. Richter, M. Sferrazza, Z. W. Grabowski, P. J. Daly, R. Broda, B. Fornal, I. Ahmad, M. P. Carpenter et al., Phys. Lett. B 336, 308 (1994). 